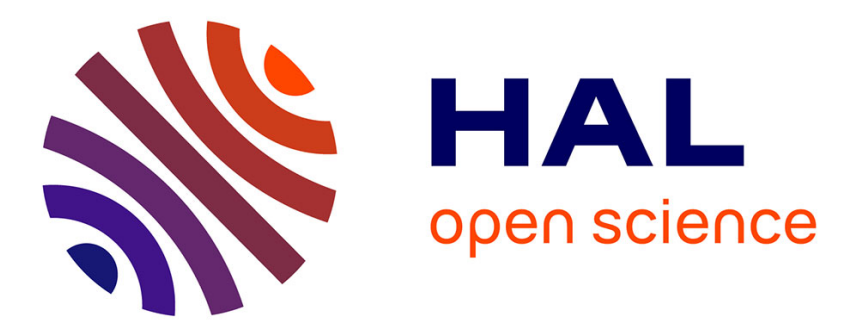

\title{
Generation of localized pulses from incoherent wave in optical fiber lines made of concatenated Mamyshev regenerators
}

\author{
Stéphane Pitois, Christophe Finot, Lionel Provost, D.J Richardson
}

\section{To cite this version:}

Stéphane Pitois, Christophe Finot, Lionel Provost, D.J Richardson. Generation of localized pulses from incoherent wave in optical fiber lines made of concatenated Mamyshev regenerators. Journal of the Optical Society of America B, 2008, 25 (9), pp.1537-1547. 10.1364/JOSAB.25.001537 . hal00406220

\author{
HAL Id: hal-00406220 \\ https://hal.science/hal-00406220
}

Submitted on 16 Apr 2010

HAL is a multi-disciplinary open access archive for the deposit and dissemination of scientific research documents, whether they are published or not. The documents may come from teaching and research institutions in France or abroad, or from public or private research centers.
L'archive ouverte pluridisciplinaire HAL, est destinée au dépôt et à la diffusion de documents scientifiques de niveau recherche, publiés ou non, émanant des établissements d'enseignement et de recherche français ou étrangers, des laboratoires publics ou privés. 


\title{
Generation of localized pulses from incoherent wave
}

\author{
in optical fiber lines
}

\section{made of concatenated Mamyshev regenerators}

\author{
Stéphane Pitois* and Christophe Finot \\ Institut Carnot de Bourgogne (ICB), UMR-CNRS 5209, Université de Bourgogne, \\ 9 Avenue Alain Savary, 21078 Dijon, France
}

\author{
Lionel Provost, David J. Richardson \\ Optoelectronics Research Centre (ORC), University of Southampton, Southampton SO17 1BJ, \\ United-Kingdom \\ *Corresponding author: spitois @u-bourgogne.fr
}

In this work, we investigate the novel properties of optical fiber lines made of Mamyshev regenerators based on self-phase modulation and subsequent spectral filtering. In particular, we show that such a regenerator line can be used to generate random sequences of optical pulses from an incoherent wave. This behaviour is related to the existence of stable eigen-pulses which can propagate unchanged through the regenerator line and act as attractors for incoming pulses. By changing the regenerator parameters, we also report the existence of multiple eigen-pulses and limit-cycles. Finally, we demonstrate that Mamyshev's regenerators could be used as efficient nonlinear gates in fiber laser cavities.

OCIS codes: $190.5530,190.3100,070.4340,320.7140$ 


\section{Introduction}

Propagation of light in optical fiber lines with periodically inserted nonlinear regenerating devices has undoubtedly attracted much attention these last ten years. Such an interest has been stimulated by the development of new techniques enabling all-optical regeneration at bit rates up to $160 \mathrm{Gbit} / \mathrm{s}$ in optical fiber transmission systems. Indeed, a variety of methods, including saturable absorbers [1], nonlinear optical loop mirrors (NOLMs) [2] or soliton reshaping [3] have been proposed to combat the cumulative impairments occuring during the propagation of picosecond pulses in fiber links.

Beside these applications, it has also been demonstrated that more fundamental phenomena can be observed in fiber transmission lines with periodically inserted nonlinear devices. A typical example is the theoretical prediction $[4,5]$ and experimental observation [6] of autosolitons in fiber lines including nonlinear optical loop mirrors. In this context, the term "autosoliton" describes robust localized pulses which can propagate in the system thanks to an equilibrium condition between nonlinearity, dispersion, amplification and nonlinear dissipation.

In the present paper, we study an optical fiber line made of concatenated Mamyshev regenerators [7]. Let us recall that Mamyshev's optical Regenerator $(M R)$ relies on the SPMinduced spectral broadening of the degraded signal followed by subsequent offset spectral filtering. Given the ease of implementation, numerous works have theoretically and experimentally focused their attention on the performance of such regenerators to efficiently restore the quality of corrupted data streams for telecommunication applications [7-15]. For example, its capacity in terms of multi-wavelength regeneration [16] or its potential miniaturisation thanks to photonic waveguides [17] are among the latest reported achievements. 
In a recent article [18], we got interested in the asymptotic properties of a transmission line made of a large number of concatenated double-stage MRs. Adopting this rather different perspective has allowed us to highlight fundamental properties of MRs such as the existence of particular eigen-pulses preserving both phase and intensity profiles along the line. In this present work, we further investigate these fundamental features and propose some possible new applications which illustrate novel properties of a line of MRs.

This paper is organised as follows. After having first reviewed the operating principle of the Mamyshev regenerator, the model we used and the nature of the eigen-pulses, we will investigate the impact of the regenerator parameters. In the second part of this paper, we will focus on the asymptotic evolution of an incoherent input signal and will outline and show that the temporal distribution of the resulting pulses is not purely random. Some original applications dealing with telecommunication signals will then be detailed. Multiple eigenpulses and limit cycles will also be considered in part 6 of this work. Finally, we will extend our conclusions to a laser geometry, leading to the demonstration of a potentially new modelocking technique.

\section{Model and principle}

\subsection{System}

The system under consideration is represented in Fig. 1 and is formed by the concatenation of $K$ identical elementary optical blocks. Each elementary block comprises two distinct Mamyshev Regenerator stages. In a first stage, light is amplified in order to reach a peak power $P_{\text {in }}$ at the input of a normally dispersive nonlinear fiber. We assume that the optical fiber has a positive second order dispersion $\beta_{2}$, a Kerr nonlinearity $\gamma$ and a fiber length $L$. 
During propagation in this fiber, the pulses undergo a large spectral broadening, mainly due to self-phase modulation (SPM). An optical band-pass Gaussian filter (OBPF1), with a 1/e half spectral width $\delta f$ and detuned by a frequency offset $\Omega$ from the initial frequency $\omega_{0}$, is then used to select a part of the broadened spectrum. As a result, a large part of the energy is lost from the system and only pulses with a high peak power can transmit a part of their energy after filtering. We would like to emphasize that this nonlinear dissipation is a key element of the physical system considered here. A second amplifier with gain $G$ is then inserted to compensate for losses associated with the previous spectral filtering. The second stage operates in a similar way as the first stage and the parameters of the second fiber are chosen to be identical to those of the first fiber. The only difference concerns the second band pass optical filter $(O B P F 2)$ which is now centered around the initial frequency $\omega_{0}$ ensuring that the whole optical block preserves the initial central wavelength.

\subsection{Model}

To simulate the optical system described above, we numerically integrate the following standard nonlinear Schrödinger equation which describes the propagation of the complex electric field $\psi(z, T)$ in the optical fibers [19]:

$$
i \frac{\partial \psi}{\partial z}=\frac{\beta_{2}}{2} \frac{\partial^{2} \psi}{\partial T^{2}}-\gamma|\psi|^{2} \psi
$$

where $T$ is the time in a copropagating time-frame and $z$ the propagation distance. For the sake of simplicity, we do not incorporate terms describing noise emitted from the amplifier, fiber losses, nor higher-order linear or nonlinear terms in our model. Note that we have checked that amplifier noise, if appropriately filtered, does not introduce qualitative changes in the asymptotic behaviour we observe [12]. We have also recently demonstrated that the 
regenerator design could be efficiently extended to take into account the distributed losses of the fiber [13].

Given the vast variety of media used to implement Mamyshev regenerators which range from $\mathrm{km}$ long dispersion shifted fibers [7] to $\mathrm{cm}$-scale long highly nonlinear chalcogenide waveguides [17], it is more convenient for a general discussion to transform Eq. (1) into the following normalized form (normally dispersive case) [19]:

$$
i \frac{\partial u}{\partial \xi}=\frac{1}{2} \frac{\partial^{2} u}{\partial \tau^{2}}-|u|^{2} u
$$

where $u, \tau$ and $\xi$ are normalized parameters defined by:

$$
u(\xi, \tau)=N_{\text {in }} \quad U, \quad U(\xi, \tau)=\frac{\psi}{\sqrt{P_{i n}}}, \quad \tau=\frac{T}{T_{0}}, \quad \xi=\frac{\beta_{2}}{T_{0}^{2}} z
$$

$P_{\text {in }}$ is the peak power of the initial pulse, whereas $T_{0}$ represents a temporal width linked to the output spectral width of the filter:

$$
T_{0}=\frac{1}{2 \pi \delta f}
$$

$N_{i n}$ is defined as:

$$
N_{i n}=\sqrt{\frac{T_{0}^{2}}{\beta_{2}} \gamma P_{i n}}
$$

Similarly, we can define $N_{\text {out }}, N$ and $N_{a v}$ as respectively:

$$
N_{\text {out }}=\sqrt{\frac{T_{0}^{2}}{\beta_{2}} \gamma P_{\text {out }}}, \quad N=\sqrt{\frac{T_{0}^{2}}{\beta_{2}} \gamma P}, \quad N_{a v}=\sqrt{\frac{T_{0}^{2}}{\beta_{2}} \gamma P_{a v}} .
$$


where $P_{\text {out }}$ is the output peak power after the propagation in the fiber and offset filtering, $P$ is the instantaneous power of the pulse and $P_{a v}$ is the initial average power of the initial pulse train considered. Normalized temporal widths $\tau_{\text {in }}, \tau_{\text {out }}$ and fiber length $\xi_{L}$ are also given by:

$$
\tau_{\text {in }}=\frac{T_{\text {in }}}{T_{0}}, \quad \tau_{\text {out }}=\frac{T_{\text {out }}}{T_{0}}, \quad \text { and } \quad \xi_{L}=\frac{\beta_{2}}{T_{0}^{2}} L
$$

with $T_{\text {in }}$ and $T_{\text {out }}$ temporal half-widths at 1/e of the input and output pulses respectively.

\subsection{Transfer functions}

Before studying the propagation of an incoherent wave in the regenerator line, we believe that some significant physical insight can be gained by considering the asymptotic evolution of a single Gaussian pulse in the system. Indeed, from the propagation of such a single pulse, one can obtain important information about the transfer function (TF) of each regenerator stage, i.e. the relationship linking input and output amplitudes: $N_{\text {out }}=f\left(N_{\text {in }}\right)$. We have evaluated this function by injecting into the regenerator a Gaussian pulse with an initial pulse width $\tau_{i n}=1$ and by recording for each $N_{i n}$, the corresponding $N_{\text {out }}$ obtained after spectral filtering. Depending on the regenerator parameters, various TF shapes can be observed [8]. In this part, we focus on the regime which exhibits a large flat plateau and which has been proven to be optimal for amplitude jitter reduction. Following previously proposed design rules [8], such a regime can be obtained for example with a regenerator based on the following configuration (designed as configuration $C 1$ ): $\xi_{L}=0.18$ and $\Omega / \delta f=6.25$. The resulting $\mathrm{TF}$ for a single stage regenerator $N_{\text {outl }}=f\left(N_{\text {in }}\right)$ is plotted in Fig. 2(a) with circles.

To compensate the inherent losses of the process, an optical amplifier with a gain $G=$ $10.6 \mathrm{~dB}$ is used before the second stage regenerator. The resulting $\mathrm{TF}$ of the block $N_{\text {out }}=$ $f\left(N_{i n}\right)$ is plotted on Fig. 2(a) using a grey line. As it can be seen, the flatness of the plateau is considerably enhanced relative to the single stage regenerator. 
We then cascade $K$ regenerative blocks and plot the resulting TF in Fig. 2(a) for $K=2$ and $K=4$ (dashed and solid lines respectively). With 2 blocks, the transfer function shape is quite close to an ideal Heavyside function and with just 4 blocks, the system becomes a perfect thresholder, rejecting Gaussian pulses with $N_{\text {in }}$ below 8.2 and transmitting pulses with a higher peak power. Using more regenerative blocks does not further change this function which can be then seen as the asymptotic TF of the system. From this TF, it can also be seen that the transmitted pulses always have the same output power $N_{\text {out }}$, whatever the value of $N_{\text {in }}$ (as long as $N_{\text {in }}$ is above the threshold).

\subsection{Eigen-pulse}

As has been seen in the previous subsection, only two different output levels can coexist for the pulses emerging from the regenerator line. Such a feature is not specific to Mamyshev regenerators and can also be observed with other $2 \mathrm{R}$ regenerators. However, as we will now see, the MR presents some specific aspects of behaviour. First, contrary to NOLMs or other fiber based regenerators, the $\mathrm{TF}$ is a function which does not only depend on the instantaneous power of the pulse but also on its temporal width and indeed on its initial chirp $[8,9,14]$. In other words, if we restrict ourselves to the consideration of transform-limited Gaussian input pulses, then each initial temporal pulse width will be associated with a TF and as a direct consequence with an asymptotic TF with a different threshold. This can be clearly observed in Fig. 2(b) where we have mapped the asymptotic output power according to the initial pulse properties (temporal width and initial amplitude). Two continuous areas can then be distinguished: in the black area, the pulse is extinguished whereas in the white area, the resulting pulse has the same output peak power, whatever the initial properties. From Fig. 2(b), we can also see that ultrashort pulses $\left(\tau_{\text {in }}\right.$ below 0.2$)$ are rejected by the system. 
If we now turn our attention to the temporal shape of the output pulse, we can see that the properties of the pulses obtained in the white area do not depend on the initial pulse properties. This is fully consistent with the use of Mamyshev Regenerator as an efficient pulse reshaper $[14,15]$. Whatever the initial temporal width or initial peak power, the pulse will asymptotically converge towards the well-defined state plotted in Fig 2(c). Note that the pulse is not transformed-limited and exhibits a nearly linear residual chirp profile combined with a Gaussian intensity profile [8]. However the most important feature here is that this particular pulse can travel unchanged through the regenerator line and thus represents a stable steady state solution of the system. Given this property and by a direct analogy with linear algebra, we have decided to use the term "eigen-pulse" to describe this pulse [18]. In this context, the white area of Fig. 2(b) can then been interpreted as a basin of attraction towards the eigen-pulse of the regenerator. It is worth noting that the eigen-pulse properties only depend on the regenerator properties so that the eigen-pulse can also be seen as a form of autosoliton [4].

\section{Influence of regenerator parameters}

In this section, we will investigate how the choice of the regenerator parameters impacts the asymptotic behaviour of the system. More specifically, we will get concern ourselves with how the choice of the TF type can affect the properties of the basin of attraction.

In the preceding section, we have chosen the regenerator parameters so that the transfer function (TF) exhibits a large plateau which leads asymptotically to a Heavyside TF. At this stage, we would like to emphasize that the operating regime of the optical regenerator - or equivalently the fiber parameters and the offset filter position, have a substantial influence on the asymptotic behaviour. Indeed, by changing the regenerator parameters, other TF shapes can be obtained, for example a strictly increasing TF or a TF exhibiting an 
oscillatory structure [8]. This last kind of TF can be achieved by reducing both the spectral offset and the fiber length. For example, using $\xi_{L}=0.06, \Omega / \delta f=3.3$ and an intermediate gain $G=7.4 \mathrm{~dB}$ (configuration C2), we can obtain the single stage TF plotted in Fig. 3(a) with circles. The two-stage TF (grey solid line) is compared with the results of the direct composition of two single stages TF (dashed line). The difference illustrates the fact that the Mamyshev regeneration not only impacts the intensity of the pulse, but also its shape and that the complete description of its behaviour can not be done by considering its TF in isolation [14]. In other words, given the fact that the TF strongly depends on the input pulse, it would be wrong to consider only the composition of $\mathrm{N}$ identical $\mathrm{TF}$ for characterising the asymptotic behaviour.

From Fig. 3(b), we can see that the oscillatory structure is accentuated when the number of regenerators is increased. After only $K=4$ double stage regenerators, does the TF become very close to the asymptotic TF, i.e. a TF exhibiting two distinct levels. But contrary to the TF presented in the previous section, one notices immediately that the TF is no longer a Heavyside function but converges towards a more complex function, with the presence of some dips.

Such a transfer function shape leads to significantly different basins of attraction as illustrated in Fig. 3(c) where black inclusions are now present inside the white basins of attraction. We can also compare the results obtained with this configuration with another configuration where the intermediate gain $G$ has been slightly changed to $G=7 \mathrm{~dB}$ (configuration C3, identical to the configuration used in [18]). The global map plotted in Fig. 3(d) is quite different and reveals that the asymptotic results can be highly influenced by the precise values of the regenerator parameters, in the present case, the gain $G$. One can also make out that the basin of attraction of configuration C3 (white area) is narrower and is fully 
included in the basin of attraction of configuration $\mathrm{C} 2$. We have checked that using lower intermediate gain results in even narrower attraction basins.

\section{Evolution of an incoherent wave}

\subsection{Principle}

We now focus our attention on the evolution of an incoherent input wave in the regenerator line. To this end, we consider that the input laser source is a stochastic field of zero mean that obeys Gaussian statistics. More specifically, we consider that the incoherent laser spectrum has a Gaussian intensity profile associated with a purely random phase. The spectral shape can therefore be written as :

$$
F(v)=\exp \left[-\left(v / v_{0}\right)^{2}+i \phi(v)\right]
$$

where $v_{0}$ corresponds to a spectral intensity bandwidth of $3.3 \delta f$ and $\phi(v)$ is uniformly distributed random spectral phase. Note that the initial bandwidth was chosen to be of the order of the spectral offset value, so that even with moderate broadening in the fiber, a significant part of the input energy can pass through the first optical band pass filter within the line. However, the most important feature is that such an incoherent signal exhibits multipeaked structure so that one can expect that the portions of signal with small peak powers will vanish when propagating in the MR line whereas peaks with higher power will convergence towards the eigen-pulse of the system. This intuitive point of view is confirmed in Fig. 4 where we have plotted the evolution of an incoherent input signal with a initial coherence time of the order of $2 T_{0}$ (Fig. $4 a$ ) after propagation through $K=20$ stages. As expected, we observe a sequence of well-defined picosecond pulses (Fig. 4b), each one corresponding to the eigen-pulse of the system. Another important feature is that the number and the temporal localization of the outcoming pulses are intrinsically seeded by the random intensity and 
phase fluctuations of the initial incoherent source and are thus highly erratic.

Another practical way to visualize the convergence of the incoherent wave towards a train of well-defined pulses is proposed in Fig. 5 where we have mapped the properties of the pulses obtained at different stages in the space $\left[\tau_{\text {out }}, N_{\text {out }}\right]$. After a single regenerator (Fig. $5(a))$, we still have a cloud of points indicating that the wave is still highly erratic in nature. But after only two MR (Fig 5(b)), a clear reshaping of the pulse is observed as highlighted by the vertical localization of the points around the expected nominal value of $\tau_{\text {out }}$. After two double regenerators, the cloud clearly becomes concentrated around the properties of the eigen-pulses (Fig. 5(c)). Finally, after only $K=40$ stages, all the pulses have all the same properties, as outlined by the single point visible in Fig. 5(d)

\subsection{Influence of the input power and of the transfer function}

We now investigate the influence of the initial average power of the incoherent wave launched into the regenerator chain. More precisely, we have counted the number of pulses emerging from the MR line for a given time frame of $1000 T_{0}$. The results are plotted in Fig. 6(a) with circles for configuration $\mathrm{C} 1$. We can make out three parts in this evolution: for low average power $\left(N_{a v}<4\right)$, no pulse are generated as the power is insufficient to fall into the basin of attraction. Then, the number of generated pulses increases in a nearly linear fashion with $N_{a v}$, indicating that more and more pulses fall into the basin of attraction. Finally, saturation occurs with around 80 pulses generated.

To better understand the temporal arrangement of the pulses, we have calculated the distribution of the time spacing between two successive pulses. A histogram of this distribution is plotted in Fig. 6(b). It is noticeable that no pulses are spaced by less than $6 T_{0}$ showing that the temporal distribution is not purely random. To interpret this spacing, we got 
studied the propagation of two Gaussian pulses spaced by $5.2 T_{0}$ in the HNLF. The longitudinal evolution of the temporal profile is plotted in Fig. 7(a). We can then see that during the propagation in this normally dispersive segment, the pulses undergo a temporal broadening which leads to pulse overlap in the edges of the pulses (what is also called in ref. [8] a collision). This overlapping creates a sinusoidal beating which can then further evolve due to nonlinear effects [20]. This severely impacts the filtered signal, leading to energy transfer from one pulse to the other $[8,14]$.

Such a progressive energy transfer between pulses can lead to the disappearance of one of the pulses, as illustrated in Fig. 7(b1) where we have considered the evolution with $K$ of the pulses at the output of the HNLF before spectral filtering. The pulses are initially spaced by $5.9 T_{0}$. As can be seen, the small energy transfer via the pulse wing increases with $K$ and finally leads to significant oscillations in the central overlap region (around $K=40$ for this given example). The nonlinear behaviour of the MR then leads to the elimination of one of the pulses.

For comparison, the evolution of two pulses spaced by $6.05 T_{0}$ is plotted in Fig. 7(b2) and with such a configuration, the effects of the overlapping is far less pronounced so that the pulse can propagate unaffected by the neighbouring pulse. We thus have here an explanation of the temporal minimal asymptotic spacing observed between adjacent pulses in the incoherent input case. Given this minimal spacing between consecutive pulses, it is also then clear that the number of generated pulses will saturate for a given time window.

We have also investigated the influence of the regenerator parameters. We tested the configuration $\mathrm{C} 2$ and $\mathrm{C} 3$ and the results are plotted in Fig. 6(a) using diamonds and triangles respectively. We can then see that the number of generated pulses is reduced compared to the results obtained with configuration $\mathrm{C} 1$. This can be explained by looking at the area of the basin of attraction which is wider for $\mathrm{C} 1$ than for $\mathrm{C} 2$ and $\mathrm{C} 3$. Similarly, many more pulses are 
generated using $\mathrm{C} 2$ than $\mathrm{C} 3$, in agreement with the results plotted in Figs. 3(c-d). We can also make out, for example for $\mathrm{C} 3$, than the evolution is not continuous. This results from the fact that the basin of attraction is not continuous and contains parts where the pulse asymptotically vanishes. Concerning the minimal time separation observed in configuration $\mathrm{C} 2$, we can see from Fig. 6(c) that slightly closer pulses can be obtained. This is linked to the shorter piece of HNLF fiber used for this configuration, which results in less overlap between successive pulses.

\section{Various applications to pulse train generation}

In the previous part, we have shown that it is possible to generate a train of well-defined pulses with undefined time spacing from an incoherent input light wave. In this part, we focus on the generation of a random train of regularly spaced pulses from a clock signal. In order to link our results to the telecommunication field, we consider a configuration $\mathrm{C} 1$ compatible with $10 \mathrm{Gbit} / \mathrm{s}$ repetition rate. The initial pulses are Gaussian pulses with a pulse width $T_{\text {in }}=12 \mathrm{ps}$, the in-line Gaussian filters have a spectral width at half-maximum equal to 40 $\mathrm{GHz}$ and the fiber length is $L=1240 \mathrm{~m}$ with a second order dispersion $\beta_{2}=6.37 \times 10^{-3} \mathrm{ps}^{2} \cdot \mathrm{m}^{-1}$ and a nonlinearity $\gamma=13 \times 10^{-3} \mathrm{~W}^{-1} \cdot \mathrm{m}^{-1}$.

The working power of the regenerator is chosen to be on the border of the attraction basin plotted in Fig. 2(b), i.e. $N_{\text {in }}=8.12$, leading in real units to a peak power of $0.735 \mathrm{~W}$ or an average power of $94 \mathrm{~mW}$. A small amount of white noise with an average power of $0.5 \mathrm{~mW}$ is superposed on the clock signal. The resulting initial pulse train is plotted in Fig. 8(a1). The amplitude noise is sufficient to make some pulses switch from the basin of attraction to the vanishing part of the map. The results obtained after $K=1$ and $K=10$ regenerators are plotted in Figs. 8(a2) and 8(a3), respectively. We can clearly see that the resulting train becomes random. If we now study the respective eye-diagrams obtained by this 
technique (see Figs. 8(b)), we can see that the small amplitude jitter present in the clock signal disappears in the asymptotic train. However, one can see some residual timing jitter in the asymptotic train and which is inherent to the Mamyshev technique itself [14].

In Fig. 8(c), we have presented some statistics concerning the number of pulses having a given peak power during the propagation through the MR line. At the input of the line, the peak powers of the pulses are localized around $0.735 \mathrm{~W}$ (see Fig. 8(c1)). After a transition stage (Fig. $8(c 2)$ ), the system reaches its equilibrium state and half of the $10 \mathrm{Gbit} / \mathrm{s}$ sequence is occupied by zero bits whereas the other half of the sequence is occupied by the eigen-pulses of the line (Fig. 8(c3)).

Finally, we would like to emphasize that the number of "ones bits" in the final sequence can easily be controlled by changing the value of the average input power. Figure 9 illustrates this property by showing the evolution of the numbers of "ones" and "zeros" in the output sequence as a function of the input power and for a constant amount of noise.

Another interesting application could be the generation of optical packets of pulses. Such a result can be obtained by adding a small deterministic amplitude variation such as a sinusoidal intensity modulation to the clock signal. A typical example is illustrated in Figs. 10: Figs. 10(a) and 10(b) represent the initial pulses sequence at $10 \mathrm{GHz}$ with an applied sinusoidal intensity modulation of 6.4 ns periode, whereas Figs. 10(c) and 10(d) show the square optical packets generated after just $K=10$ stages. Note that the temporal widths of these packets can easily be adjusted by changing the average power of the input sequence.

For the two preceding applications, we have used as an initial signal a signal based on a clock train. But we can also easily adapt the proposed device in order to deal with a sinusoidal signal made by beating together two continuous wave signals. As a result of the 
nonlinear propagation in the presence of normal dispersion, the initial beat signal will progressively reshape and additional harmonics will be generated through self-phase modulation [21]. As the initial shape is no longer Gaussian, the operating power has to be adapted [22] so that we now used $P_{\text {in }}=1.43 \mathrm{~W}$ in our simulations. The results are plotted in Fig. 11 and show that a random train of eigen-pulses with a fixed spacing is efficiently generated. 


\section{Mutliple eigen-pulses and limit cycles}

In the preceding sections, we have considered systems exhibiting only one eigen-pulse. But for other sets of parameters, we have also observed that the regenerator line reveals more complex behaviour. Beginning from configuration $\mathrm{C} 1$, we now change the length ratio $\xi_{L}$ and we measure the number of output powers that are asymptotically obtained. These results are plotted in Fig. 12. We can see that for $\xi_{L}$ above 0.075 , only one output level is recorded. But, for a lower ratio, this number increases, indicating the presence of multiple eigen-pulses. For $\xi_{L}$ between 0.06 and 0.075 , two states are visible, and this number will grow when $\xi_{L}$ decreases. Fig. 12 can then be seen as a bifurcation towards chaos.

\subsection{Multiple eigen-pulses}

In this subsection, we focus on the region where two eigen-pulses can coexist. To illustrate our conclusions, let us focus on the particular value of $\xi_{L}=0.0649$. We have plotted the two invariant solutions in Fig. 13 on a linear (a) and logarithmic scale (b). We can then see that the two eigen-pulses differ not only in their output amplitude, but also in their characteristic shapes, the wings of the pulses exhibiting some slight differences. As a consequence, each pulse will be associated with its own transfer function, as shown in Fig. 13(c). The output powers are found at the crossing point between the TF and the dashed line.

Following a similar procedure to the one used in part 2, we have also mapped in Fig. 14 the attraction basins which can be expected from this regenerator. The resulting map highlights the fact that the multiple basins are distributed according to a complex pattern. We have used various scales, which also indicate that fractal structures can be observed at the borders of the basins. Similar results could be obtained with other configurations such as the one used in ref. [18] (see Fig. 4(b) of this ref.). 
Let us now consider the influence on this system of the propagation of an incoherent wave. The resulting sequence exhibits two pulses which is consistent with the existence of two eigen-pulses. This can be also clearly seen in the convergence diagram obtained as shown in Fig. 15(a) where two points can be observed after propagation through $K=80$ regenerators. However one can also see that some points fall outside those convergent points. Despite the propagation in further regenerators, such points will still asymptotically exist. Their presence is linked to the collisions occurring between pulses. Indeed, eigen-pulses 1 and 2 have different peak powers, which leads to different delays induced during propagation in the regenerator [14]. As a consequence, this induces a different velocity which leads progressively to the occurrence of a collision where the eigen-pulse 1 collides with eigenpulse 2. Such a situation is illustrated in Fig. 15(b). The collision results in perturbation of the pulses, both in terms of temporal width and amplitude as highlighted by the non-converging points.

We can also propose similar applications to those developed in section 5. For example, by taking a Gaussian pulse train with a working power chosen close to the border, the output sequence will exhibit three levels which are randomly distributed as shown in Fig. 16. Those two levels are easily seen in the eye diagrams obtained (see Fig. 16(f)). Let us however note that due to the power dependant induced delay, the central levels of the pulses are distinct.

\subsection{Limit cycles}

In Fig. 12, we have seen that the region where two levels are present is followed by a zone where four distinct levels are observed. In this section, we then focus on this aspect and try to clarify the nature of the pulses. To illustrate our study, we consider a configuration with $\xi_{L}=$ 0.0569. By comparing trains generated from an incoherent wave after $K, K+1$ and $K+2$ regenerators, we can conclude that the four levels correspond to two distinct limit-cycles with 
two states each, as presented in Fig. 17(a). Let us note that cycles with a higher number of states can also be observed, as has been reported in ref. [18] where limit cycles with three states have been described. By taking an initially incoherent wave, we can also observe the convergence towards one of the four possible states as reported in Fig. 17(b). Let us also outline that similar two-states (three-states) limit-cycles behaviours have recently been reported in normally-dispersive fiber laser configurations [23] and are usually referred in this context as period-doubling (tripling) bifurcations.

\section{Hybrid regenerator and extension to laser applications}

The concept of the NOLM [2] was previously extended to encompass amplifying sections of fiber within the device. As well as providing optical switches with low input switching powers, the resulting device, referred to as the Nonlinear Amplifying Loop Mirror (NALMs [24]), has been successfully incorporated within all fibre cavities to provide self-starting passively mode-locked laser operation $[25,26]$. In the earliest work in this area, NALM based laser cavities allowed the spontaneous formation of optical pulses from optical noise due to soliton effects within the device. Likewise it is possible to envision active variants of Mamyshev regenerators and the use of optical similariton concepts [27] to realise new passively mode-locked fiber laser systems. Pulses propagating in normally dispersive amplifying fiber experience both SPM and gain and evolve self-similarly undergoing both a temporal and spectral broadening ([27, 28]). The profile of the pulses evolves towards a parabolic form with a linear chirp. Recent examples based on Raman gain [27], erbium [29] or ytterbium [30] have highlighted that self-similar amplification is fully compatible with high pulse repetition rates.

Here, we consider such a concept based on the use of the double stage MR shown in Fig. 18. This particular configuration further shows that discrete sections of amplifying fiber and passive HNLF can be combined within a single double-stage device to obtain the desired 
pulse formation properties. We have chosen to focus on ytterbium doped fibers, but similar results could have been demonstrated with erbium [28] or Raman amplifiers [31]. The optical amplifying fiber we used has a constant linear gain $g=12.5 \mathrm{~dB} \cdot \mathrm{m}^{-1}$, a normal dispersion $\beta_{21}=$ $3510^{-3} \mathrm{ps}^{2} \cdot \mathrm{m}^{-1}$, a non-linearity $\gamma_{1}=10.710^{-3} \mathrm{~W} \cdot \mathrm{m}^{-1}$ and a length $L_{1}=2 \mathrm{~m}$. The optical filter used has a spectral bandwidth $\delta f=1 \mathrm{THz}$ and is shifted by $\Omega=1.8 \mathrm{THz}$. 3-dB insertion losses are taken into account for the filters. The second regenerator is more traditional and based upon a single mode fiber with normal dispersion at $1060 \mathrm{~nm}\left(L_{2}=3 \mathrm{~m}, \gamma_{2}=6.410^{-3} \mathrm{~W} \cdot \mathrm{m}^{-1}\right.$, $\left.\beta_{22}=3010^{-3} \mathrm{ps}^{2} \cdot \mathrm{m}^{-1}\right)$. An optical attenuator ( $4 \mathrm{~dB}$ loss) is inserted in order to have a convenient working power.

Propagation in the amplifier is simulated using the nonlinear Schrödinger equation with a constant gain term [28]. For the sake of simplicity, we have not included in our simulation pump depletion, gain saturation or the finite bandwidth of the amplifier. In addition we assume the use of polarization maintaining fibers so that the effects of nonlinear polarization rotation can be neglected.

The transfer function obtained after filtering of the output pulses is plotted in Fig. 18(b). As it can be seen, the device exhibits a flat transfer function. Similar behaviour is observed as in case of a symmetric double stage regenerator operating with a single eigenpulse, meaning that the conclusions of our previous studies can be extended to this hybrid regenerator with two highly asymmetric single stage regenerators. The eigen-pulse of the device is in plotted Fig. 18(c).

Extension of this system to the fiber laser domain is quite straightforward. The principle of the fiber cavity model is sketched in Fig. 19(a). We have also plotted in Fig. 19(b) the evolution of the peak-power and temporal-width of the pulse through a cavity roundtrip. The self-similar evolution of the pulse inside the YDFA is evidenced by the combined growth of the peak-power and the temporal width. This evolution significantly differs from the 
dynamics observed in the passive segment where the temporal broadening of the pulse leads to a decrease of the peak power. From Fig. 19(b), it is also clear that the highest pulse energy is obtained before the OBPF 1 . We therefore introduce a coupler to extract $20 \%$ of the cavity energy (OA value is adapted to $3 \mathrm{~dB}$ loss). The resulting output pulses are shown in Fig. 19(c) and clearly exhibit the characteristic temporal profile of a parabolic pulse. Their nearly linear chirp can be compensated by use of a pair of diffraction gratings so that sub-ps pulses are readily achievable (see Fig. 19(d)).

We thus consider that we have demonstrated a new way to efficiently modelock a fiber laser with the double stage MR acting both as a saturable absorber and an optical limiter. The proposed system thus constitutes a new kind of all-normal dispersion fiber laser - a topic which has recently attracted significant interest (see [23, 32] and references within).

\section{Conclusion}

In this work, we have used a novel approach to highlight the novel properties of all-optical regenerators based on self-phase modulation and subsequent spectral filtering. It is shown that such regenerators can be used to generate random sequences of optical pulses from an incoherent wave. This behaviour is related to the existence of eigen-pulses which can propagate unchanged through the regenerator line and act as attractors for incoming pulses. Such a property could be of a particular interest for the design and optimisation of doublestage Mamyshev's optical regenerators for optical telecommunications applications. In particular we envisage the extension of our results to the formation of eigen-states in optical transmission lines which have the potential to lead to significant improvements in system performance [4]. We also would like to point out that randomly distributed pulses may find numerous applications beyond the field of telecommunications. For example, in the field of radar-type applications, random pulse trains can be used to generate electromagnetic impulses 
at random intervals, thus greatly reducing susceptibility to system detection by undesirable external sources. In biological systems, synaptic input impulses (involved for example in artificial neural networks) can be modelled by such random pulses trains comprising pulses with approximately equal amplitude and duration. Finally, we would like to state that such optical pulse trains, including random bits sequences, can be used to perform autocorrelationbased distance measurements.

In a second aspect of this work, we also demonstrate that multiple eigen-pulses and limitcycles can be obtained by changing the regenerator parameters. It is anticipated that the underlying properties and reasons for such behaviour would form the subject of interesting future investigations enabled by a more systematic study of the system parameters. Finally, our results indicate that Mamyshev regenerators can represent efficient nonlinear gates for use in fiber lasers providing novel means to achieve passive mode-locking.

\section{Acknowledgment}

We would like to acknowledge J.H.V. Price for fruitful discussions. 


\section{References}

1. J. Fatome, S. Pitois, A. Kamagate, G. Millot, D. Massoudre, and J. L. Oudar, "Alloptical reshaping based on a passive saturable absorber microcavity device for future $160 \mathrm{~Gb} / \mathrm{s}$ applications," IEEE Photon. Technol. Lett. 19, 245-247 (2007).

2. $\quad$ N. J. Doran and D. Wood, "Nonlinear-optical loop miror," Opt. Lett. 13, 56-58 (1988).

3. M. Asobe, A. Hirano, Y. Miyamoto, K. Sato, K. Hagimoto, and Y. Yamabayashi, "Noise reduction of $20 \mathrm{Gbit} / \mathrm{s}$ pulse train using spectrally filtered optical solitons," Electron. Lett. 34, 1135-1136 (1988).

4. S. Boscolo, S. A. Derevyanko, S. K. Turitsyn, A. S. Kovalev, and M. M. Bogdan, "Autosoliton propagation and mapping problem in optical fiber lines with lumped nonlinear devices," Phys. Rev. E 72, 016601 (2005).

5. V. S. Grigoryan, "Autosoliton in a fiber with distributed saturable amplifiers," Opt. Lett. 21, 1882-1884 (1996).

6. A. Gray, Z. Huang, Y. W. A. Lee, I. Y. Khrushchev, and I. Bennion, "Experimental observation of autosoliton propagation in a dispersion-managed system guided by nonlinear optical loop mirrors," Opt. Lett. 29, 926-928 (2004).

7. P. V. Mamyshev, "All-optical data regeneration based on self-phase modulation effect," in European Conference on Optical Communication, ECOC'98, 1998), 475476.

8. L. Provost, C. Finot, K. Mukasa, P. Petropoulos, and D. J. Richardson, "Design scaling rules for 2R-Optical Self-Phase Modulation-based regenerators 2R regeneration," Opt. Express 15, 5100-5113 (2007).

9. M. Rochette, L. B. Fu, V. G. Ta'eed, D. J. Moss, and B. J. Eggleton, "2R optical regeneration : an all-optical solution for BER improvement," IEEE J. Sel. Top. Quantum Electron. 12, 736-744 (2006).

10. J. Leuthold, G. Raybon, Y. Su, R. J. Essiambre, S. Cabot, J. Jacques, and M. Kauer, "40 Gbit/s transmission and cascaded all-optical wavelength conversion over 1000 000 km," Electron. Lett. 38, 890-891 (2002).

11. M. Matsumoto, "Efficient all-optical 2R regeneration using self-phase modulation in bidirectional fiber configuration," Opt. Express 14, 11018-11023 (2006).

12. T. N. Nguyen, M. Gay, L. Bramerie, T. Chartier, and J. C. Simon, "Noise reduction in 2R-regeneration technique utilizing self-phase modulation and filtering," Opt. Express 14, 1737-1747 (2006).

13. L. Provost, C. Finot, K. Mukasa, P. Petropoulos, and D. J. Richardson, "Generalisation and experimental validation of design rules for self-phase modulation-based 2Rregenerators," in Optical Fiber Conference, OFC 2007, 2007), OThB6.

14. C. Finot, T. N. Nguyen, J. Fatome, T. Chartier, L. Bramerie, M. Gay, S. Pitois, and J. C. Simon, "Numerical study of an optical regenerator exploiting self-phase modulation and spectral offset filtering at $40 \mathrm{Gbit} / \mathrm{s}$," Opt. Commun. 281, 2252-2264 (2008).

15. B. E. Olsson and D. J. Blumenthal, "Pulse restoration by filtering of self-phase modulation broadened optical spectrum," J. Lightwave Technol. 20, 1113-1117 (2002).

16. L. Provost, F. Parmigiani, C. Finot, K. Mukasa, P. Petropoulos, and D. J. Richardson, "Analysis of a two-channel 2R all-optical regenerator based on a counter-propagating configuration," Opt. Express 16, 2264-2275 (2008).

17. V. G. Ta'eed, M. Shokooh-Saremi, L. B. Fu, I. C. M. Littler, D. J. Moss, M. Rochette, B. J. Eggleton, Y. Ruan, and B. Lutther-Davies, "Self-phase modulation-based 
integrated optical regeneration in chalcogenide waveguides," IEEE J. Sel. Top. Quantum Electron. 12, 360-370 (2006).

18. S. Pitois, C. Finot, and L. Provost, "Asymptotic properties of incoherent waves propagating in an all-optical regenerators line," Opt. Lett. 32, 3262-3264 (2007).

19. G. P. Agrawal, Nonlinear Fiber Optics, Third Edition (Academic Press, San Francisco, CA, 2001).

20. C. Finot and G. Millot, "Interactions of optical similaritons," Opt. Express 13, 58255830 (2005).

21. S. Pitois, C. Finot, J. Fatome, and G. Millot, "Generation of 20-Ghz picosecond pulse trains in the normal and anomalous dispersion regimes of optical fibers," Opt. Commun. 260, 301-306 (2006).

22. C. Finot, L. Provost, P. Petropoulos, and D. J. Richardson, "Parabolic pulse generation through passive nonlinear pulse reshaping in a normally dispersive two segment fiber device," Opt. Express 15, 852-864 (2007).

23. L. M. Zhao, D. Y. Tang, X. Wu, and H. Zhang, "Period-doubling of gain-guided solitons in fiber lasers of large net normal dispersion," Opt. Commun. in press(2008).

24. M. E. Fermann, F. Haberi, M. Hofer, and H. Hochreiter, "Nonlinear amplifying loop mirror," Opt. Lett. 15, 752-754 (1990).

25. I. N. Duling III, "All-fiber ring soliton laser mode locked with a nonlinear mirror," Opt. Lett. 16, 539-541 (1991).

26. D. J. Richardson, R. I. Laming, D. N. Payne, V. J. Matsas, and M. W. Phillips, "Selfstarting passively modelocked erbium fiber ring laser based on the amplifying sagnac switch," Electron. Lett. 27, 542-544 (1991).

27. C. Finot, S. Pitois, and G. Millot, "Regenerative 40-Gb/s wavelength converter based on similariton generation," Opt. Lett. 30, 1776-1778 (2005).

28. J. M. Dudley, C. Finot, G. Millot, and D. J. Richardson, "Self-similarity in ultrafast nonlinear optics," Nat. Phys. 3, 597-603 (2007).

29. Y. Ozeki, Y. Takushima, K. Taira, and K. Kikuchi, "Generation of $10 \mathrm{GHz}$ similariton pulses using Erbium-doped fiber amplifiers for application to multiwavelength sources," in Conference on Lasers and Electro-Optics (CLEO US), (OSA Trends in Optics and Photonics Series, 2004), paper CTuK2, 629-632.

30. P. Dupriez, C. Finot, A. Malinowski, J. K. Sahu, J. Nilsson, D. J. Richardson, K. G. Wilcox, H. D. Foreman, and A. C. Tropper, "High-power, high repetition rate picosecond and femtosecond sources based on $\mathrm{Yb}$-doped fiber amplification of VECSELS," Opt. Express 14, 9611-9616 (2006).

31. C. Finot, G. Millot, S. Pitois, C. Billet, and J. M. Dudley, "Numerical and experimental study of parabolic pulses generated via Raman amplification in standard optical fibers," IEEE J. Sel. Top. Quantum Electron. 10, 1211-1218 (2004).

32. F. W. Wise, A. Chong, and W. H. Renninger, "High-energy femtosecond fiber lasers based on pulse propagation at normal dispersion," Laser \& Photonics Rev. 2, 58-73 (2008). 


\section{Figures captions}

1. Schematic diagram of the regenerator line and parameters used.

2. (a) Normalized transfer function after a single stage MR (circles), a double stage MR (grey solid line), two regenerative blocks (dashed line) and after four double stage MRs (solid black line). The dotted line represents $\mathrm{N}_{\text {out }}=\mathrm{N}_{\text {in }} / \sqrt{G}$. (b) Asymptotical $\mathrm{N}_{\text {out }}$ of a Gaussian pulse propagating in the line as a function of its initial temporal width $\tau_{\text {in }}$ and initial amplitude $\mathrm{N}_{\text {in }}$. White-shaded area: the pulse converges towards $\mathrm{N}_{\text {out }}=3.27$, black-shaded area: the pulse is attenuated and finally suppressed. (c) Temporal properties of the eigen-pulse.

3. (a) Normalized transfer function after a single stage MR (circles). The TF obtained after a double stage MR (grey solid line) is compared to the combination of two single stage TF (dashed line). The dotted line represents $\mathrm{N}_{\text {out }}=\mathrm{N}_{\mathrm{in}} / \sqrt{G}$.

(b) Normalized transfer function after two regenerative blocks (dashed line) and after four double stage MRs (solid black line). (c-d) Asymptotical $\mathrm{N}_{\text {out }}$ of a Gaussian pulse propagation in the line as a function of its initial temporal $\tau$ width and initial power $\mathrm{N}_{\text {in }}$. White-shaded area: the pulse converges towards $\mathrm{N}_{\text {out }}=$ 5, black-shaded area: the pulse is attenuated and finally suppressed. Different intermediate gains are tested (c) $\mathrm{G}=7.4 \mathrm{~dB} \quad$ (d) $\mathrm{G}=7 \mathrm{~dB}$

4. Evolution of an incoherent wave with the parameters of configuration C1. (a) input, (b) for $\mathrm{K}=20$. Initial average amplitude is $\mathrm{N}_{\mathrm{av}}=6.75$. 
5. Convergence of the output properties of the generated pulses. The density of the points is presented by grey scale (a) after a single stage (b) after a double stage regenerator after two double stage regenerators (e) after $K=40$ double stage regenerators.

6. (a) Influence of the initial $\mathrm{N}_{\mathrm{av}}$ of the incoherent wave on the number of pulses normalized to a time slot of $1000 \mathrm{~T}_{0}$ for various regenerator configurations: circles, diamonds and triangles are for configurations $\mathrm{C} 1, \mathrm{C} 2$ and $\mathrm{C} 3$ respectively (b) Distribution of the time spacing between to successive pulses for configuration $\mathrm{C} 1$ and for $\mathrm{N}_{\mathrm{av}}=11$, (c) same graph with the configuration $\mathrm{C} 2$

7. (a) Longitudinal evolution of the temporal pulse profile during the propagation in the highly nonlinear fiber. (b) Evolution of a pair of pulses in the MR line separated by $5.9 \mathrm{~T}_{0}$ (b1) and $\Delta \mathrm{T}=6.05(\mathrm{~b} 2)$

8. Evolution of a train of Gaussian pulses emitted at $10 \mathrm{GHz}$ with a small amount of noise with the parameters of configuration $\mathrm{C} 1$. Intensity profiles, eye-diagrams and distributions of the peak powers of the pulses in the sequence are plotted in subfigures (a), (b) and (c) respectively. Subplots (1), (2) and (3) are the results for the initial pulses, the pulses for $\mathrm{K}=1$ and for $K=10$, respectively.

9. Evolution of the numbers of «ones» (diamonds) and «zeros» (circles) in the final sequence for configuration $\mathrm{C} 1$ as a function of the input peak power. 
10. Evolution of a train of Gaussian pulses emitted at $10 \mathrm{GHz}$ with an intensity modulation with the parameters of configuration $C 1$. (a) Input and (c) for $K=10$. (b) Input and (d) $K=10$ plotted with a difference temporal range.

11. Evolution of an initial sinusoidal beating cadenced at $10 \mathrm{GHz}$ with the parameters of configuration $\mathrm{C} 1$. (a) Input, (b) for $\mathrm{K}=2$ and (c) for $\mathrm{K}=10$. (d), (e) and (f) are the corresponding eye-diagrams.

12. Evolution of the number of output states as function of the normalized length $\xi_{\mathrm{L}}$.

13. The two eigen-pulses plotted on a linear scale (a) and on a log scale (b) (maximum of the pulse normalized to 1). In dotted grey lines, eigen-pulse 1, in black solid line, eigen-pulse 2. (c) Transfer functions associated with each eigen-pulse.

14. Ouput levels according to initial pulses parameters: in black, the pulse disappears, in grey, the pulse converges to eigen-pulse 1 and in white, the pulse asymptotically converges towards eigen-pulse 2. Various magnifications are used.

15. (a) Output properties of the generated pulses after $\mathrm{K}=80$ stages. The density of the points is presented by grey scale (b) Collision of two eigen-pulses.

16. Evolution of a train of Gaussian pulses emitted at $10 \mathrm{GHz}$ with a small amount of noise with the parameters Fig. 16. (a) Input, (b) for $K=4$ and (c) for $K=10$. (d), (e) and (f) are the corresponding eye-diagrams. 
17. (a) Pair of pulses constituting the two limit cycles

(b) Convergence of the output properties of the generated pulses. The density of the points is presented by grey scale. Diagram obtained after $\mathrm{K}=80$ double stage regenerators.

18. (a) Schematic diagram of the hybrid regenerator line and parameters used. (b) Asymptotic transfer function. $\quad$ (c) Eigen-pulse of the hybrid regenerator observed.

19. (a) Schematic diagram of the proposed laser cavity.

(b) Evolution of the pulse properties in the cavity : evolution of the temporal width versus the peak power.

(c) Output self-similar pulse observed at the $20 \%$ output coupler.

(d) Compressed pulse obtained after linear chirp compensation. 


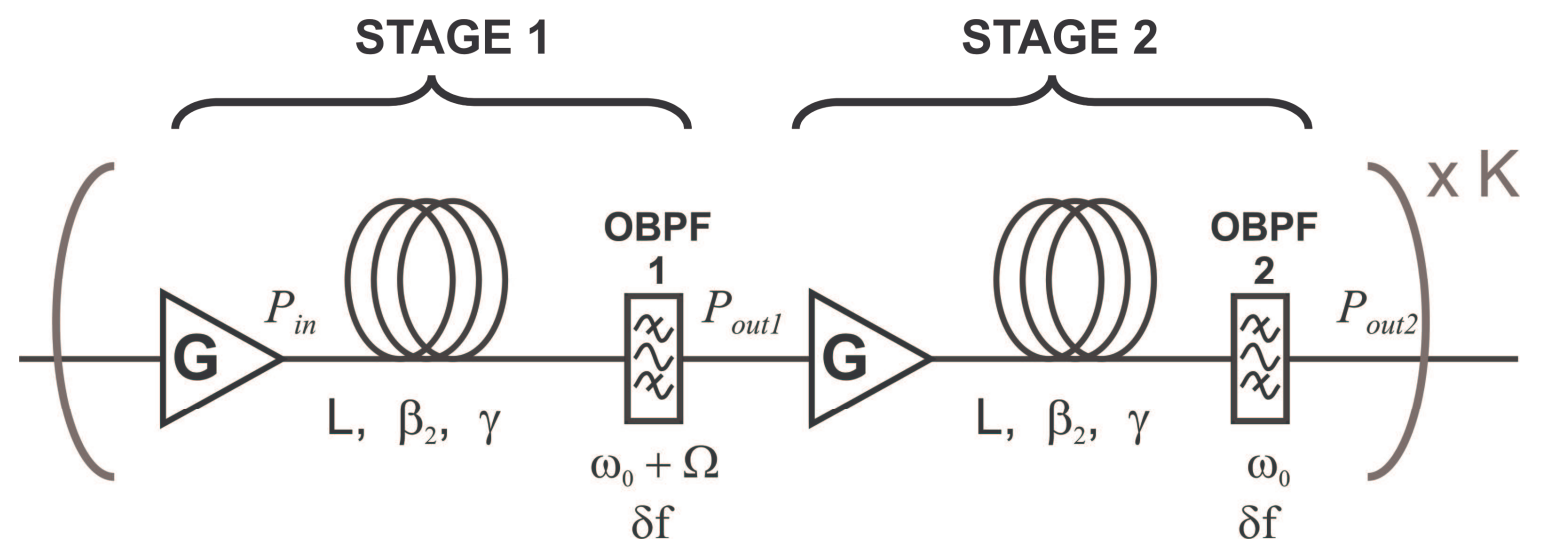

Figure 1 

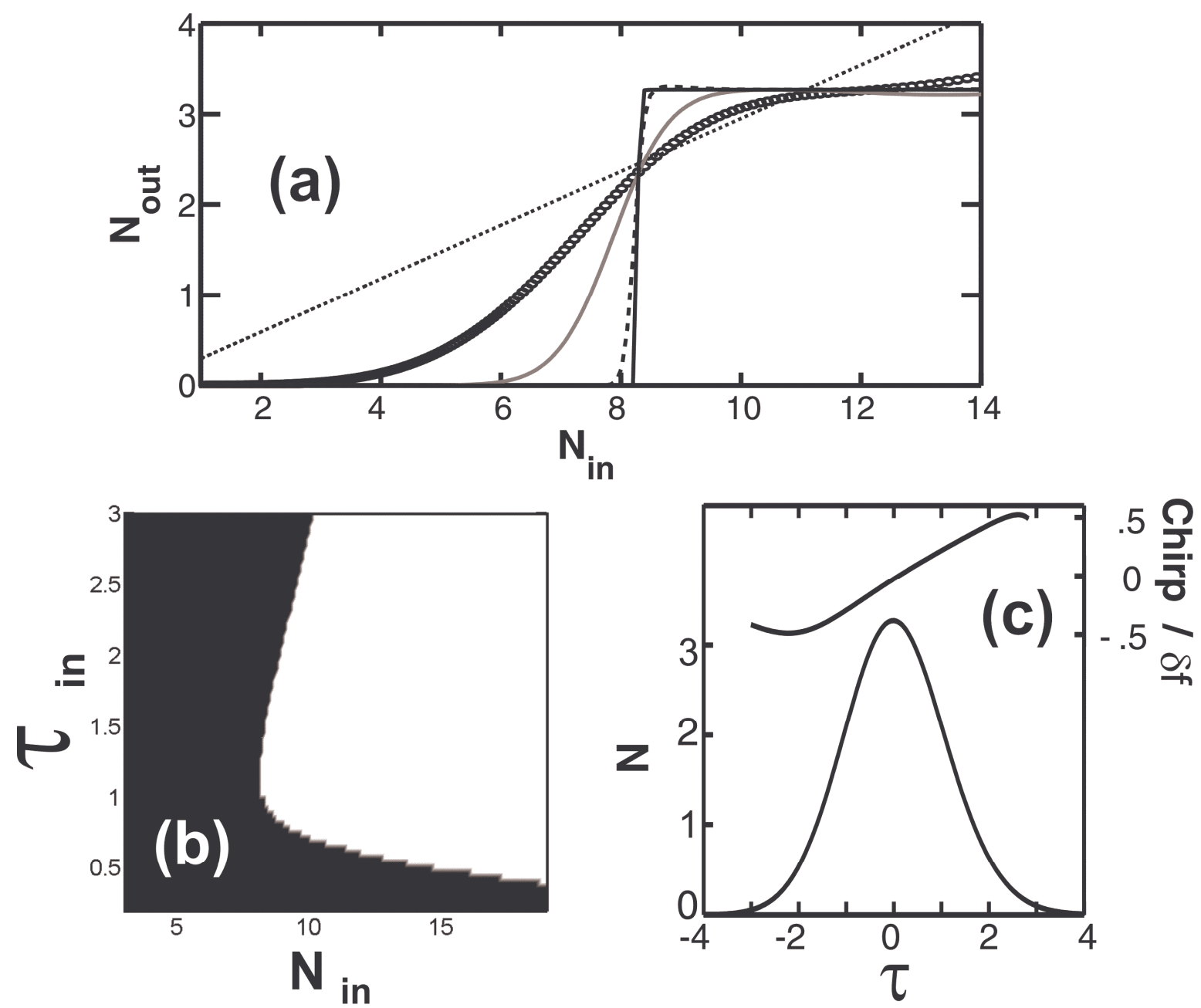

Figure 2 

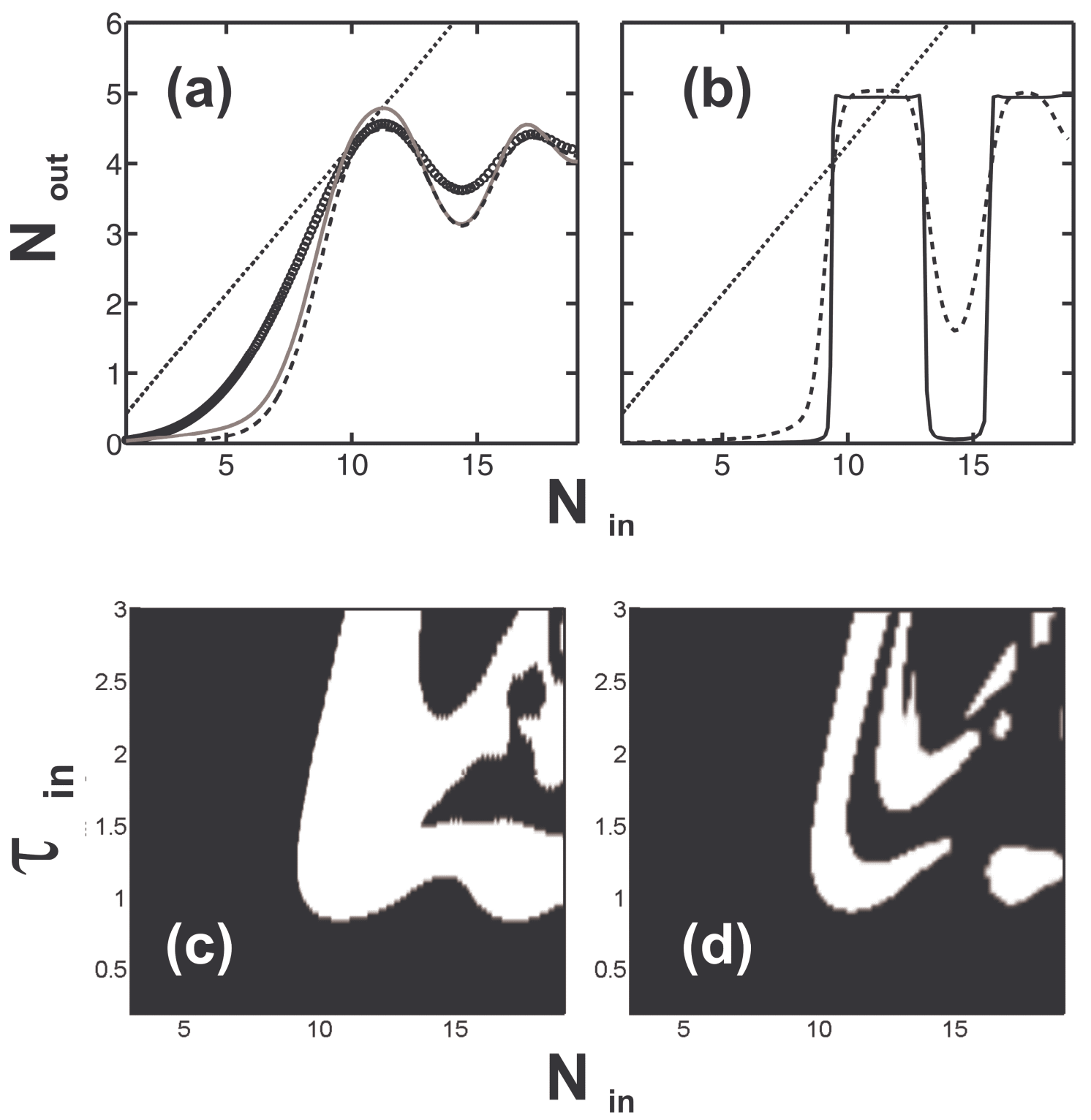

Figure 3 

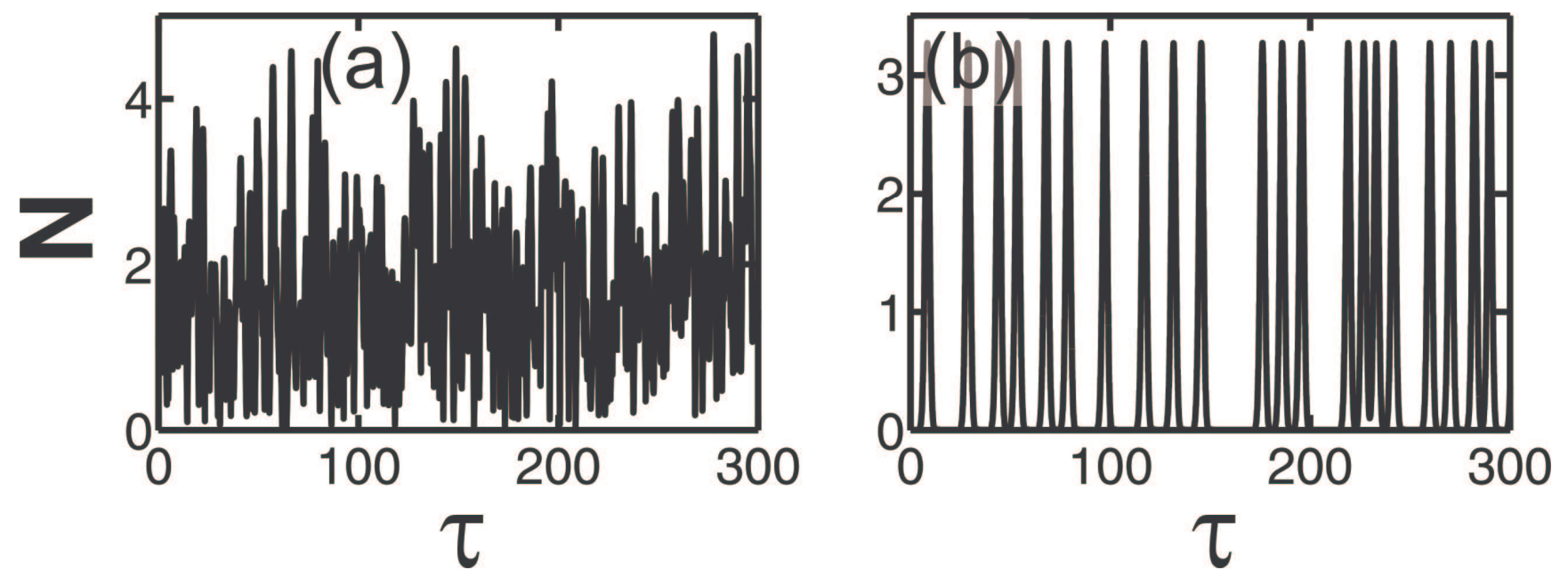

Figure 4 

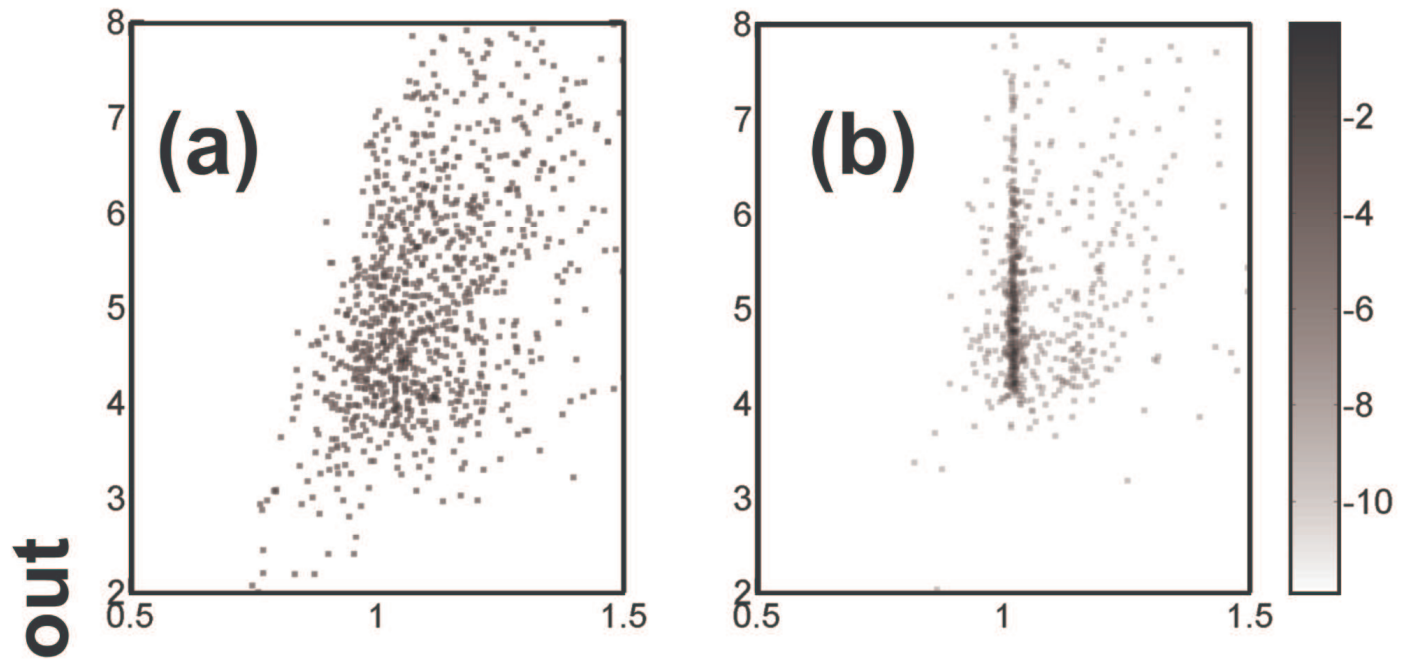

$\frac{0}{6}$
D
$\frac{0}{0}$
$\frac{5}{6}$

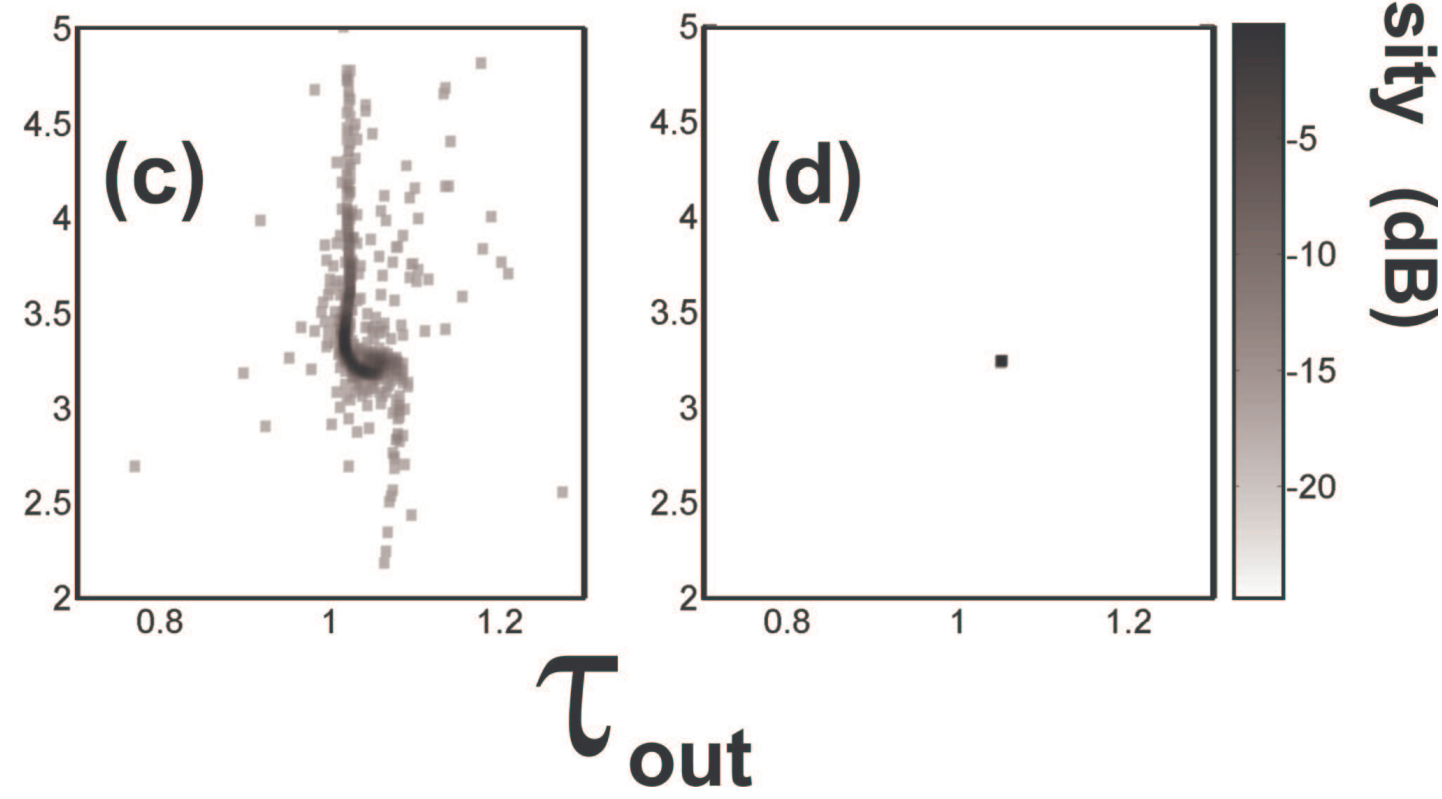

Figure 5 

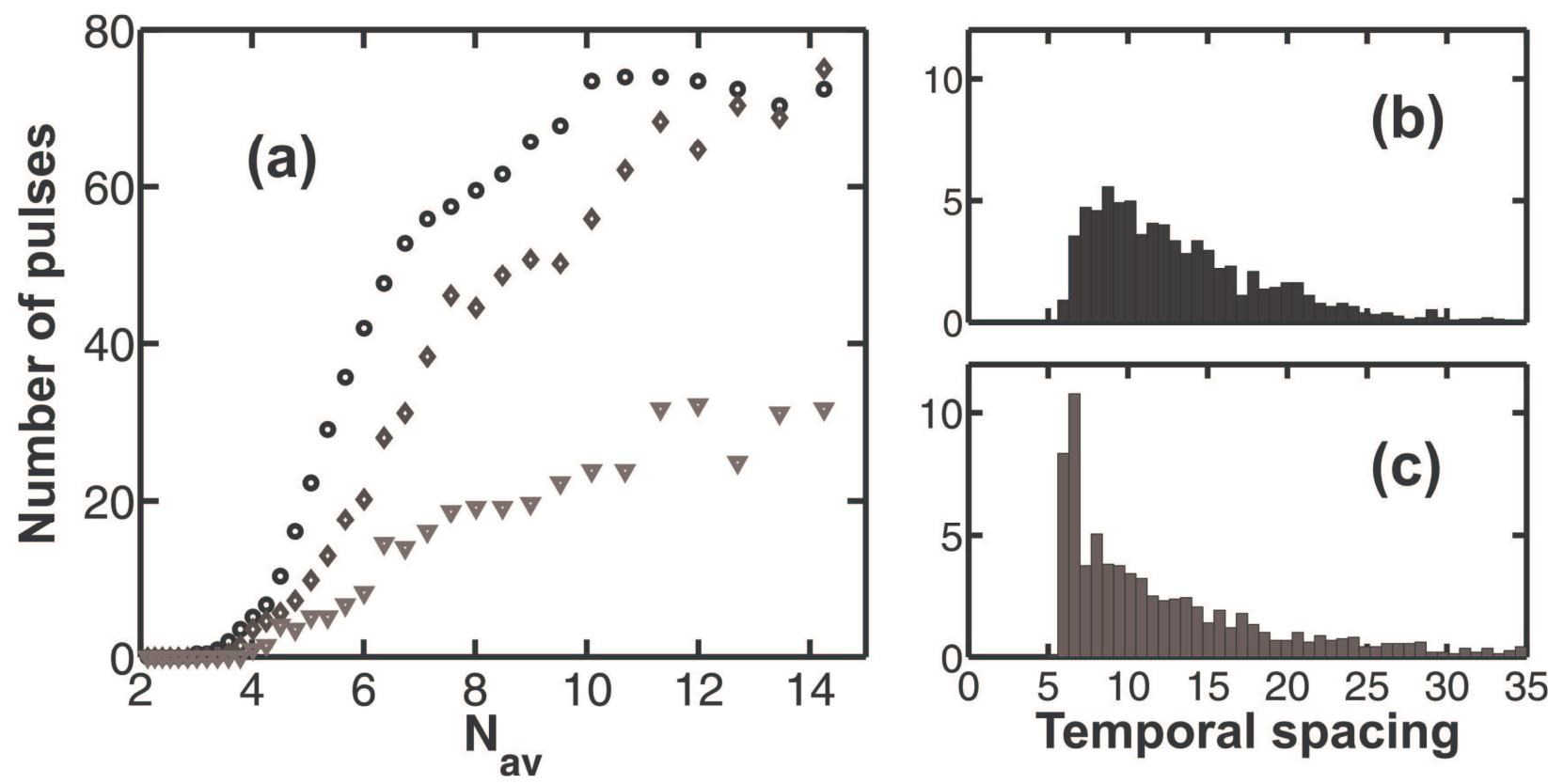

Figure 6 


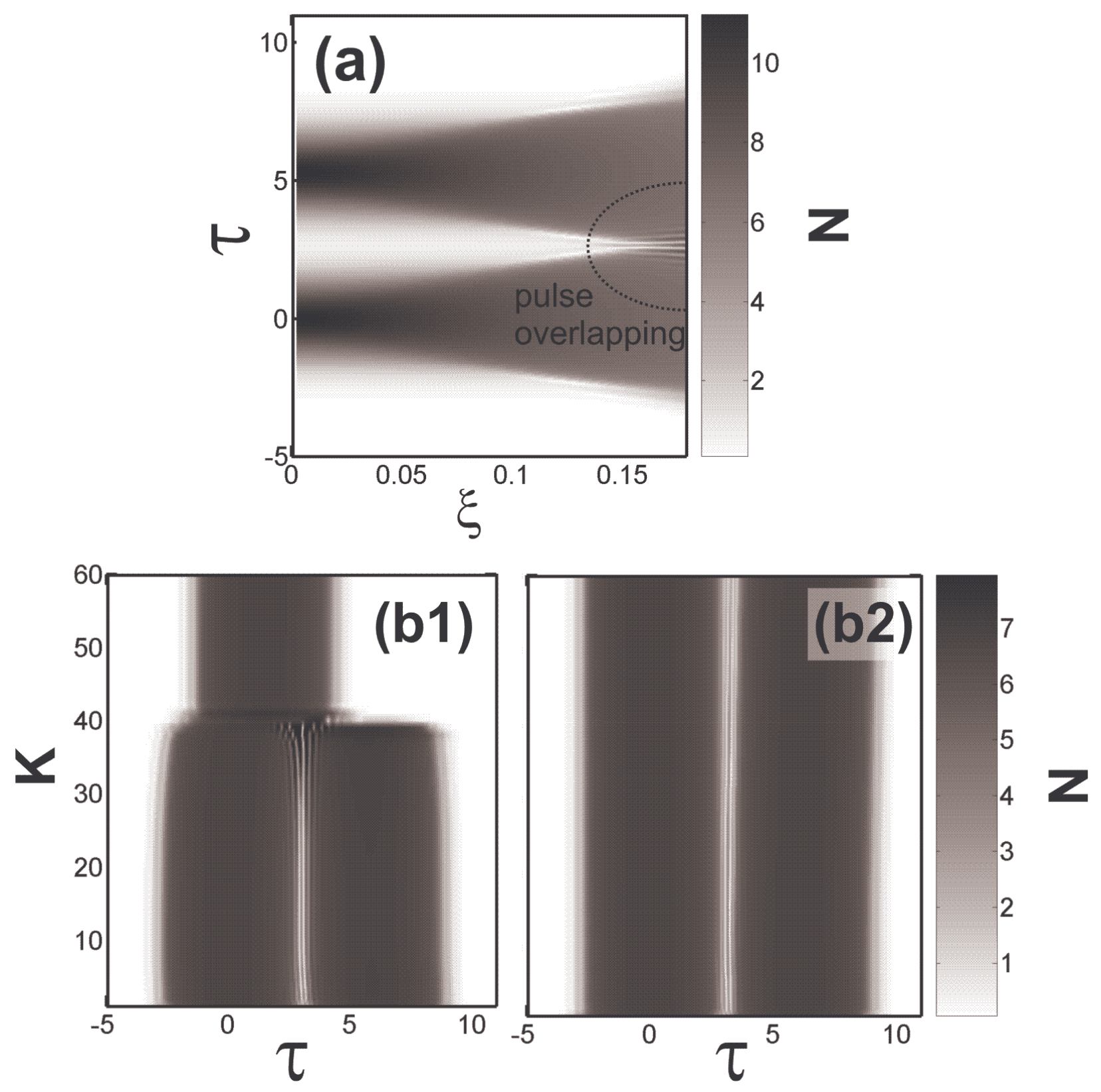

Figure 7 

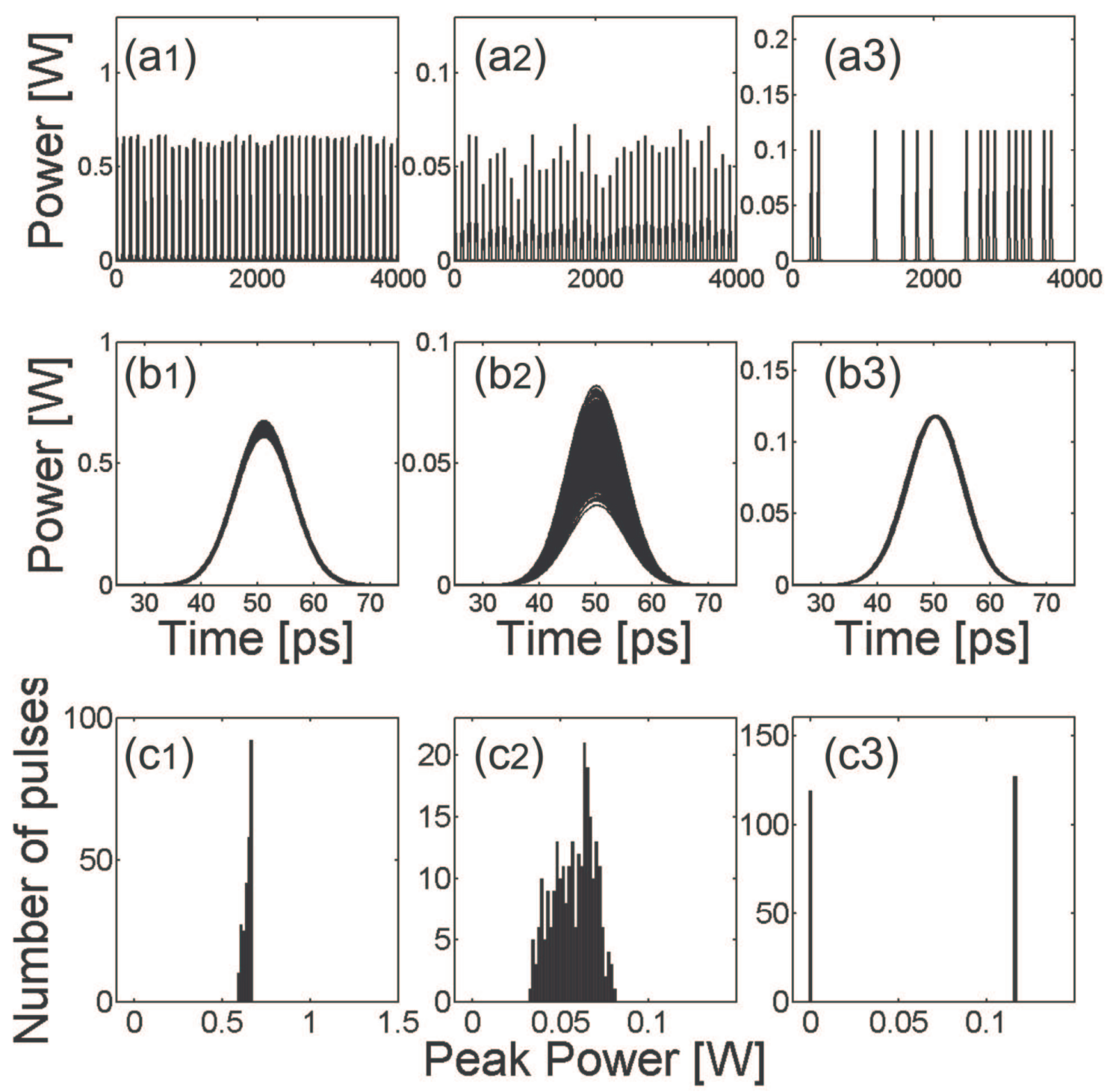

Figure 8 


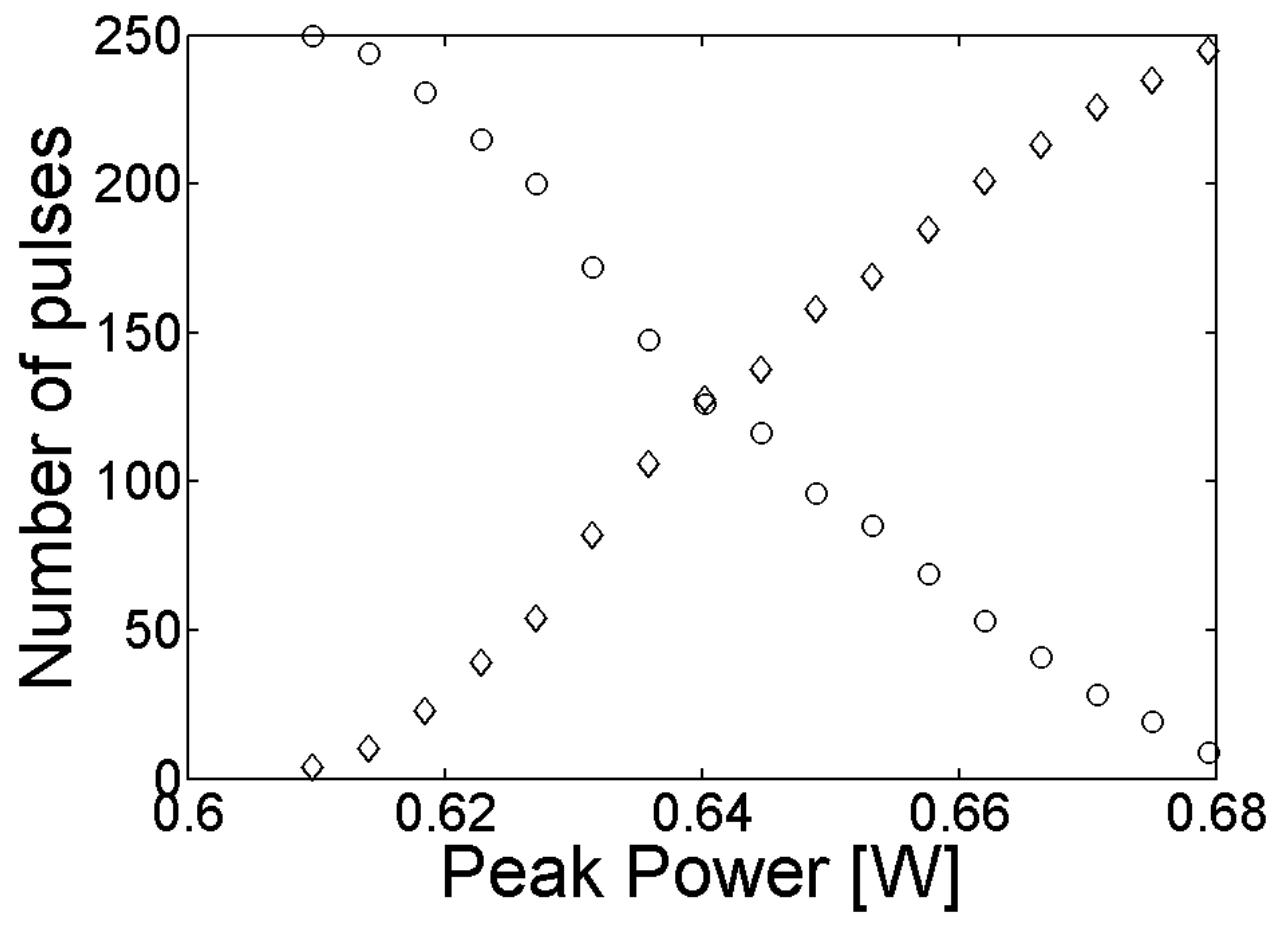

Figure 9 

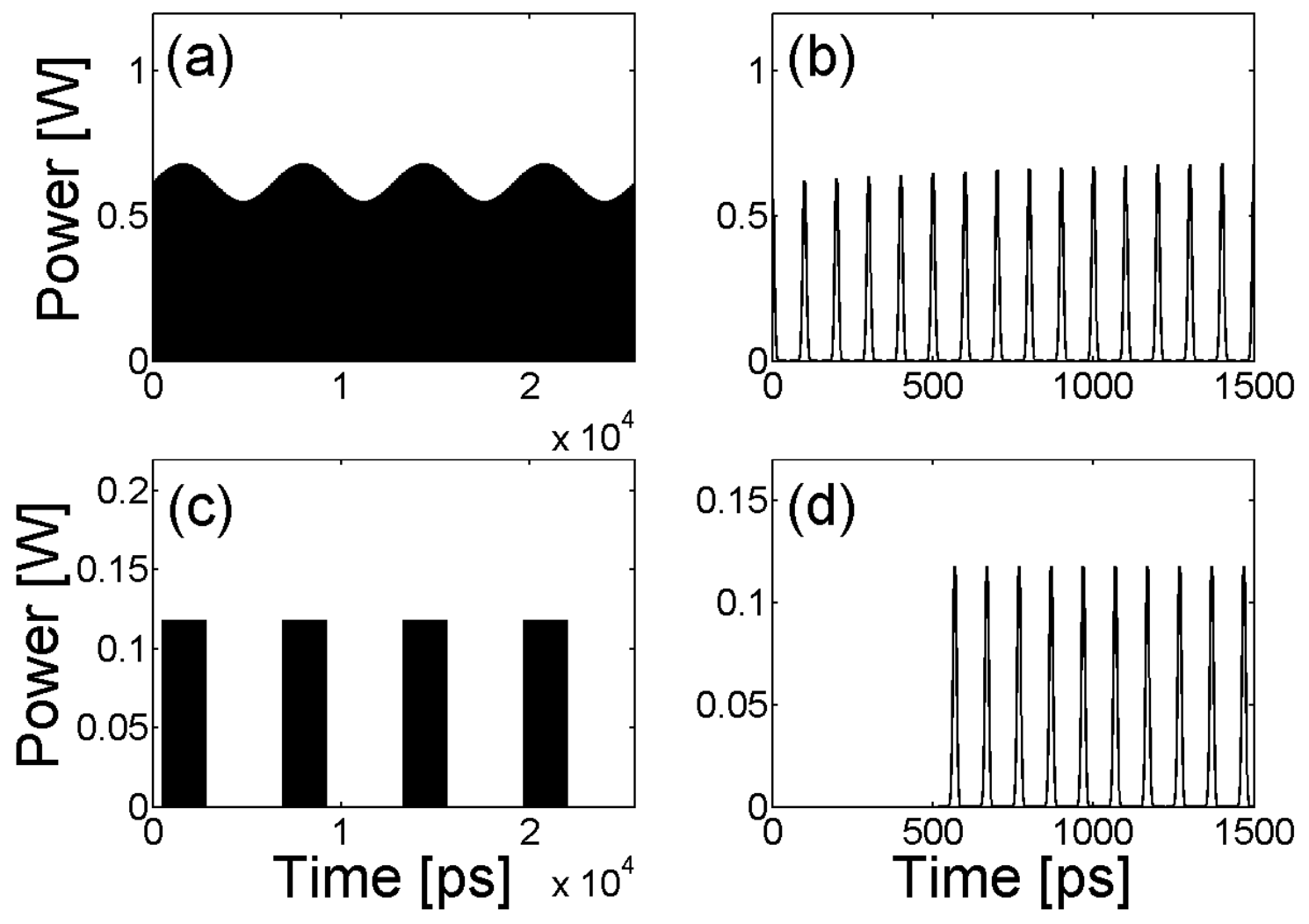

Figure 10 

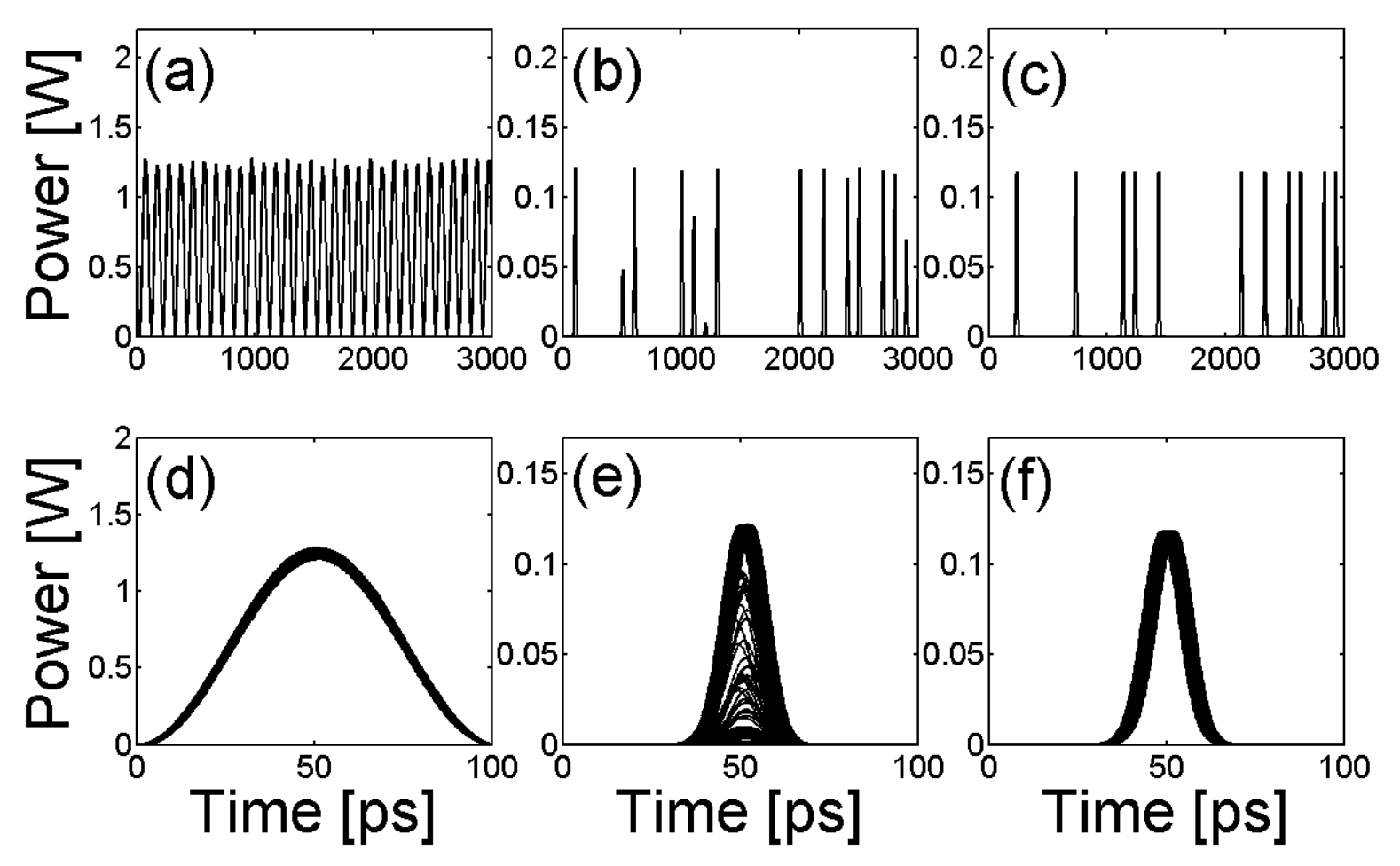

Figure 11 


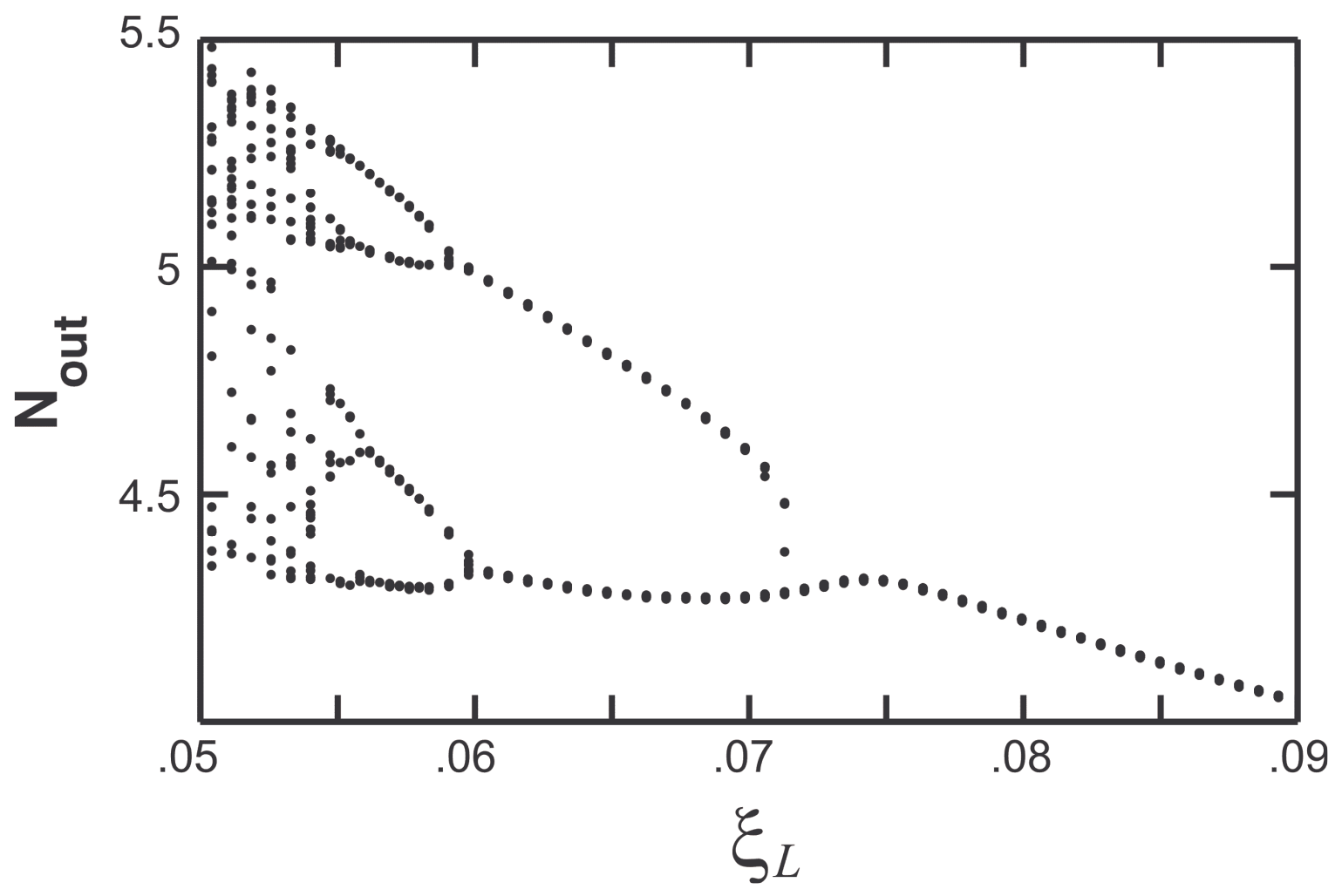

Figure 12 

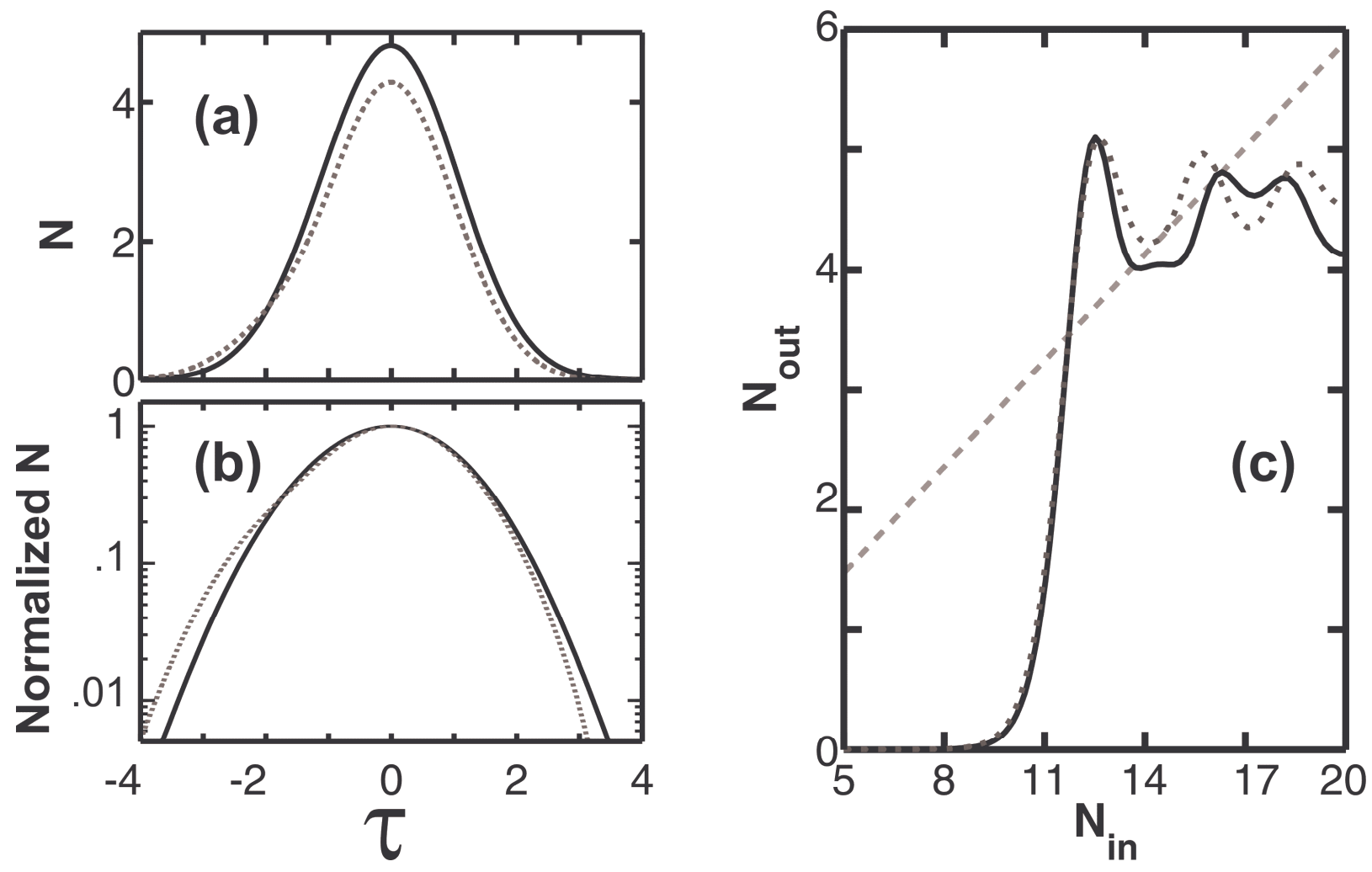

Figure 13 


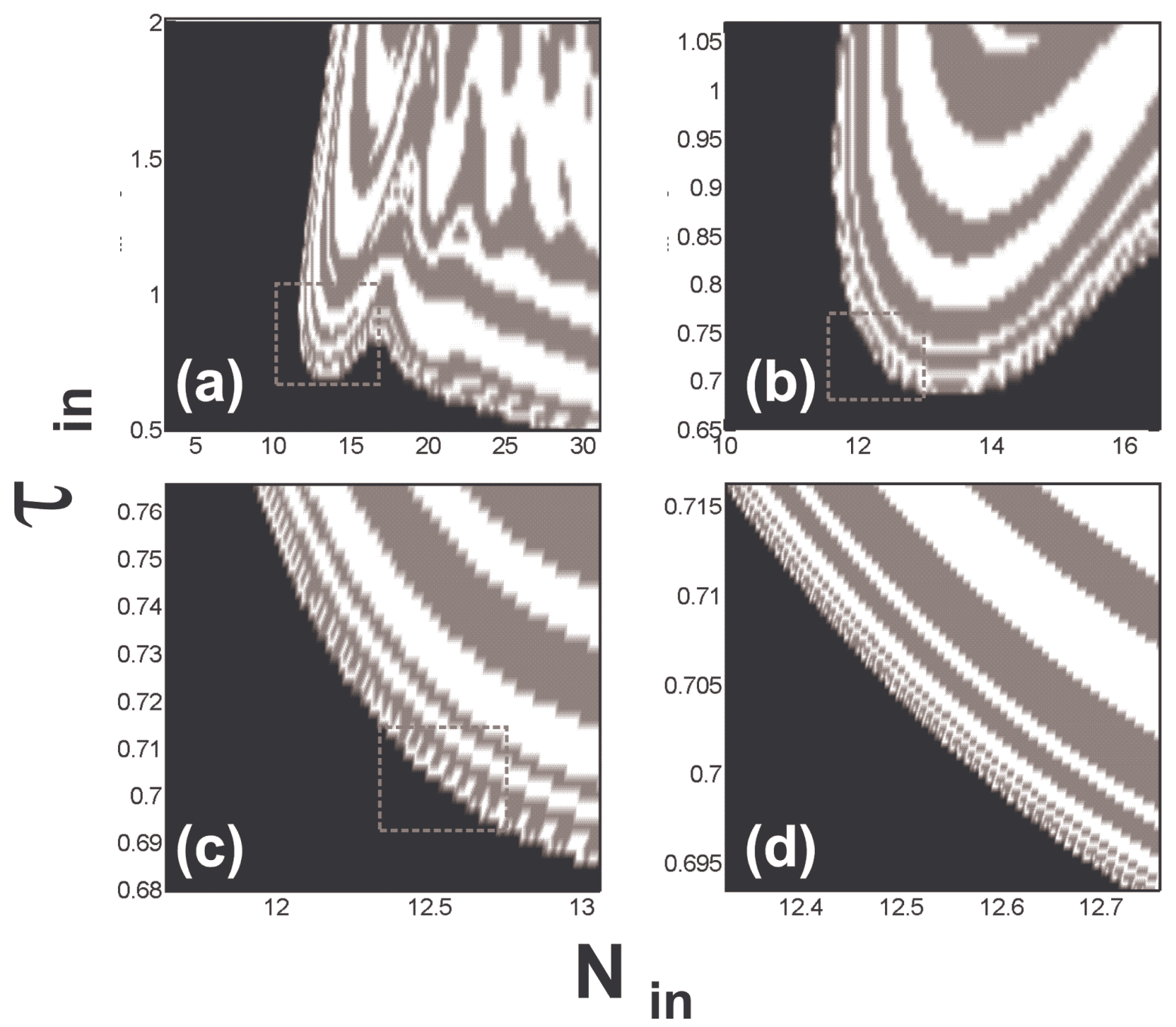

Figure 14 

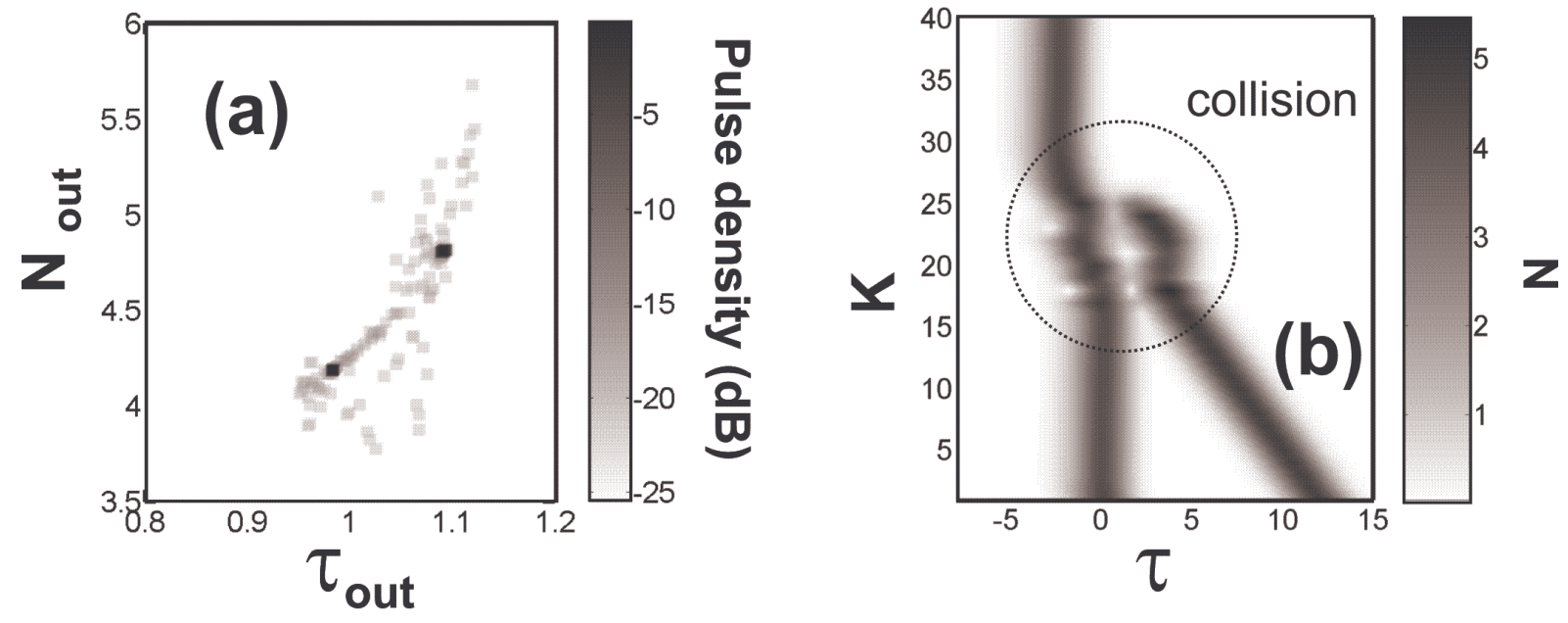

Figure 15 

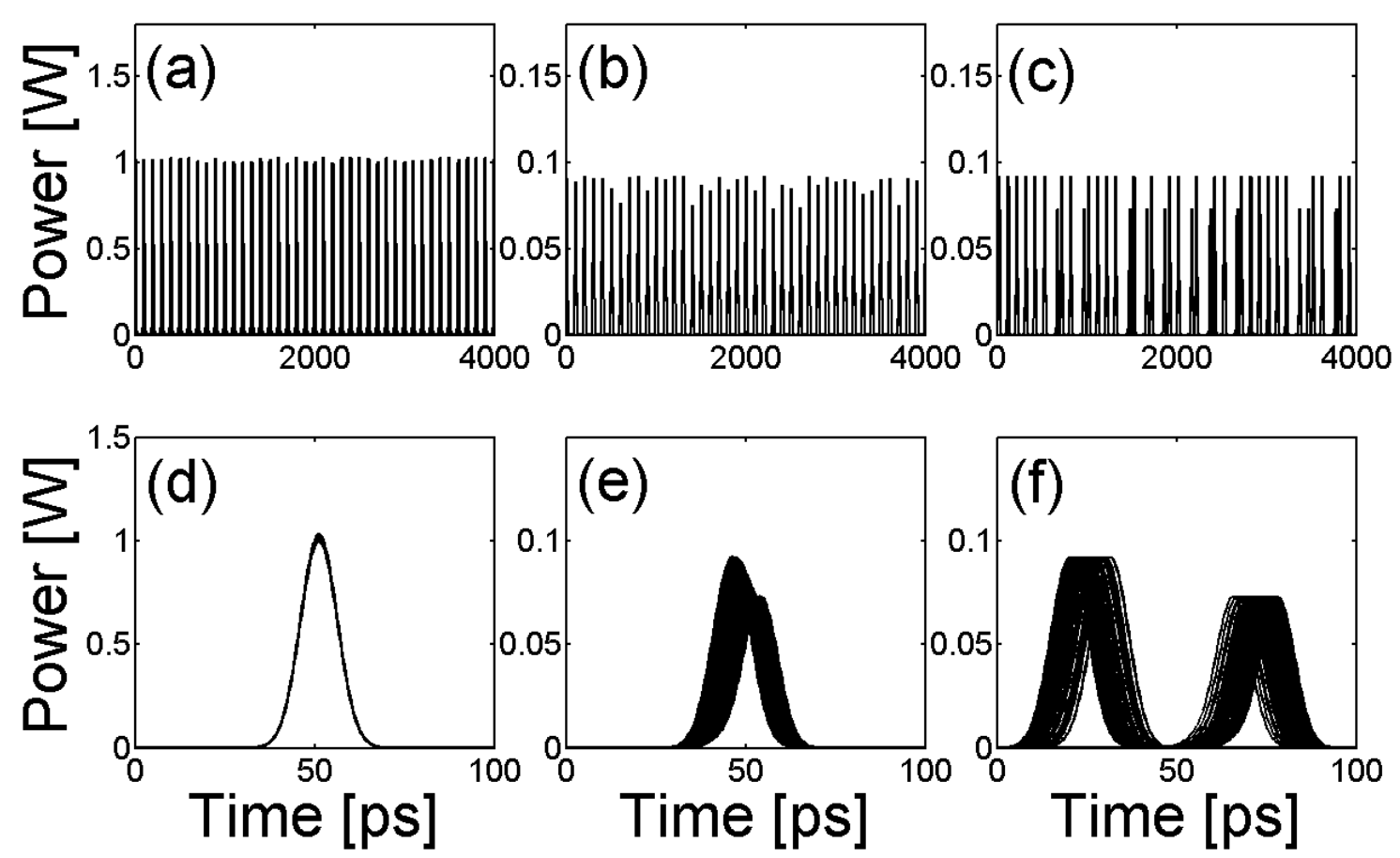

Figure 16 

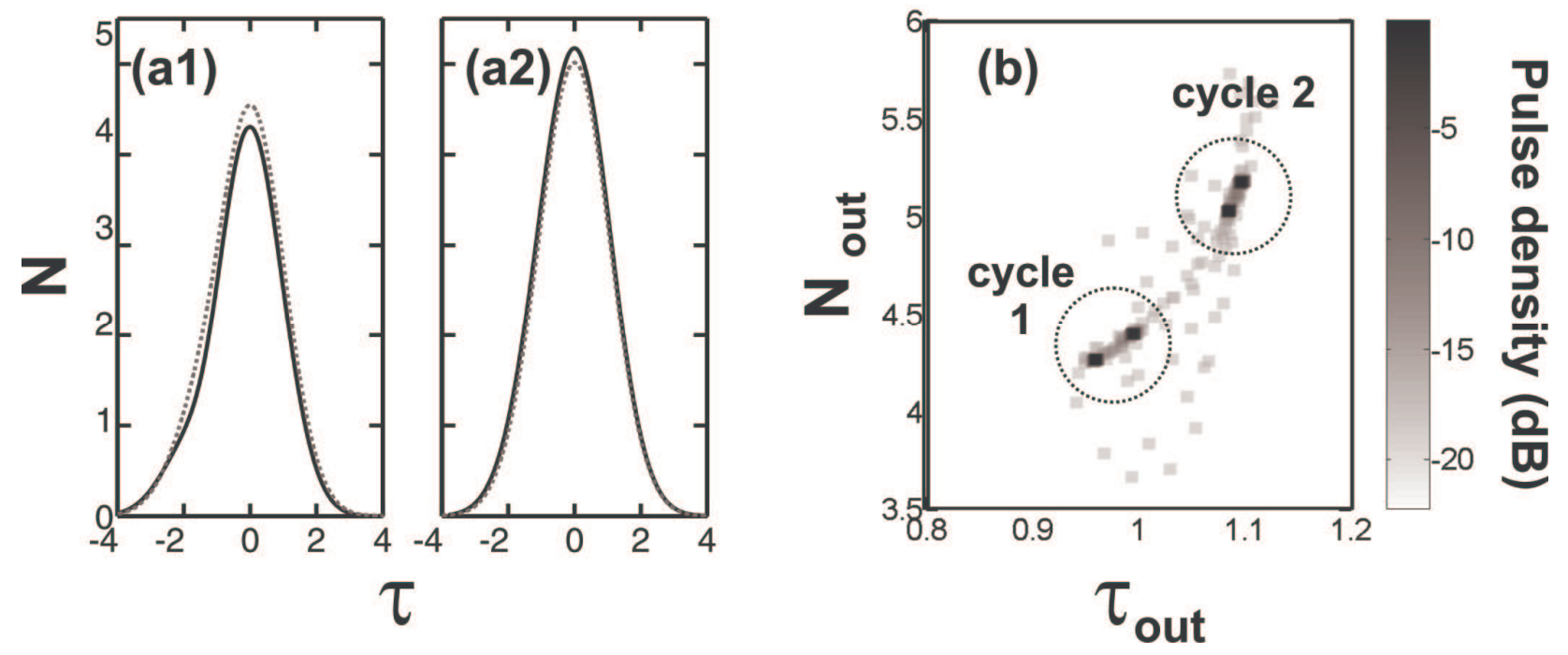

Figure 17 

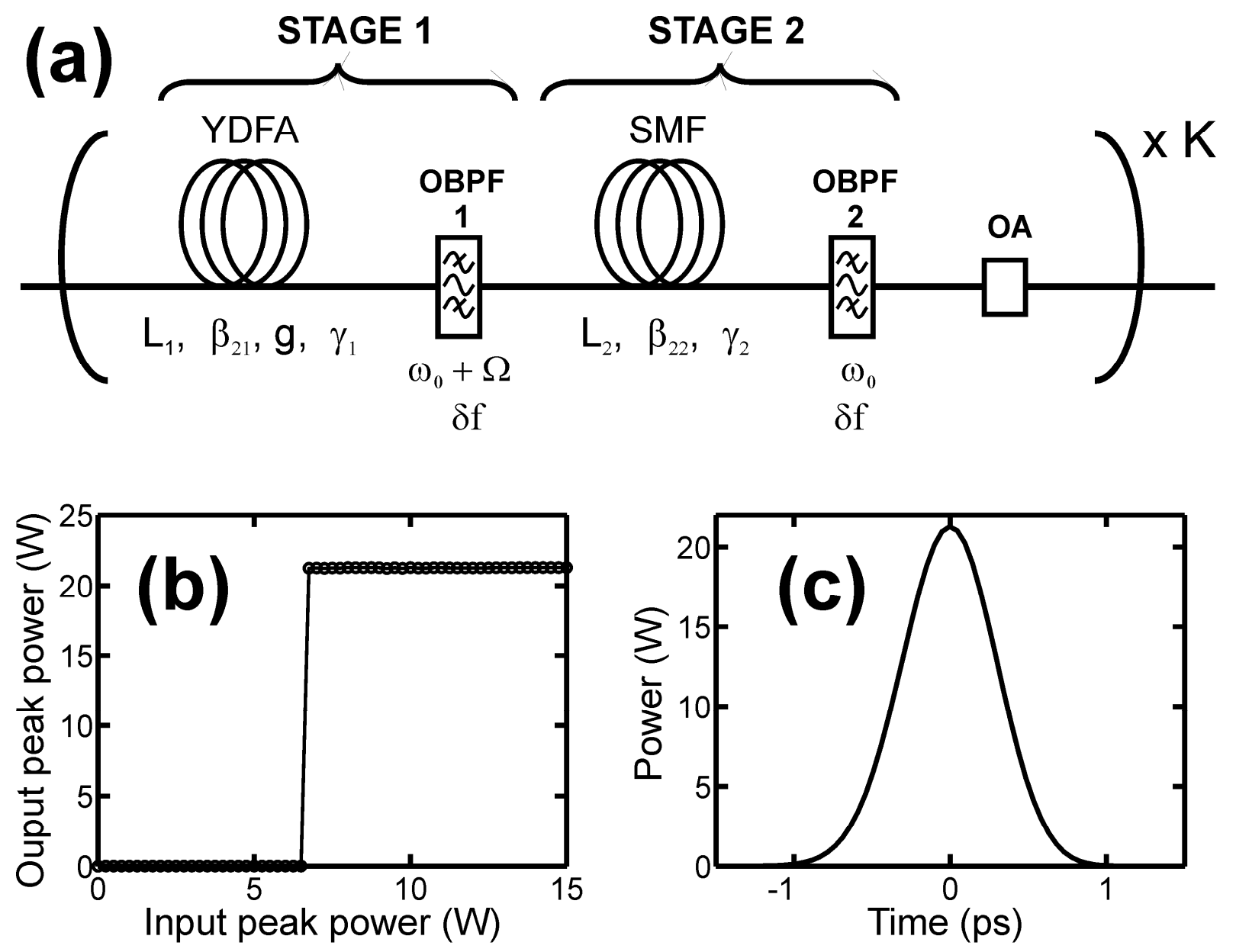

Figure 18 

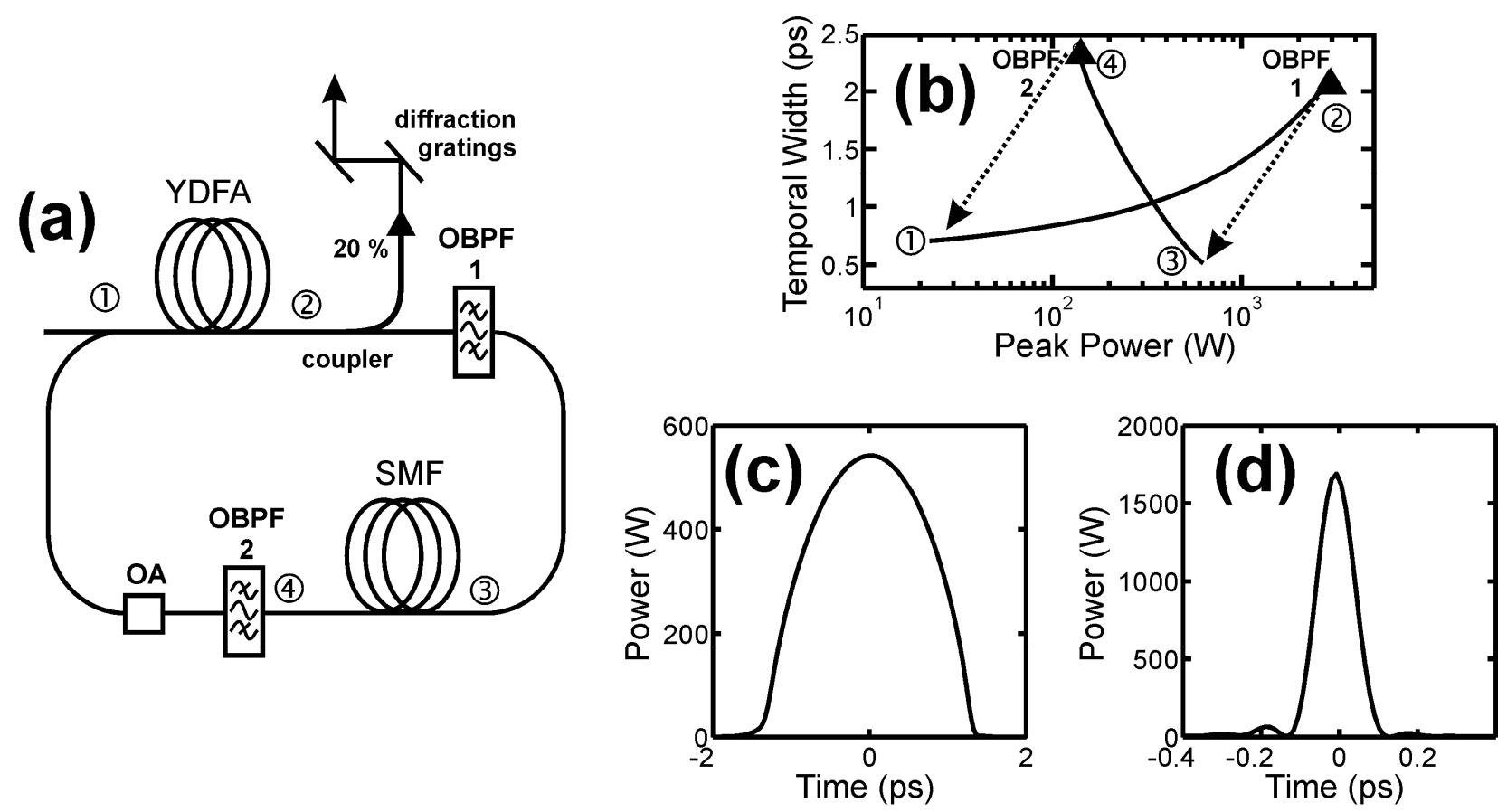

Figure 19 